\title{
The Relationship between Human Papillomavirus and Oesophageal Squamous Cell Carcinoma in China-A Review of the Evidence*
}

\author{
Surabhi S. Liyanage ${ }^{1 \#}$, Qian Li $^{2}$, Yang Zheng ${ }^{3}$, Holly Seale ${ }^{1}$, Philip J. Crowe ${ }^{4}$, Anthony T. Newall ${ }^{1}$, \\ Bayzidur Rahman ${ }^{1}$, Eva Segelov ${ }^{5}$, Chenxu Qu ${ }^{6}$, Fanghui Zhao ${ }^{6}$, Junfeng Liu ${ }^{2}$, Zhanhai Gao ${ }^{1}$, \\ Weixian Shi ${ }^{3}$, Peng Yang ${ }^{3}$, Aye Moa $^{1}$, Chandini Raina MacIntyre ${ }^{1}$
}

\begin{abstract}
${ }^{1}$ School of Public Health and Community Medicine, The University of New South Wales Medicine, The University of New South Wales, Sydney, Australia; ${ }^{2}$ Department of Surgery, Fourth Hospital, Shijiazhuang, China; ${ }^{3}$ Centre for Disease Control and Prevention, Beijing, China; ${ }^{4}$ Department of Surgery, Prince of Wales Hospital Clinical School, The University of New South Wales, Sydney, Australia; ${ }^{5}$ Department of Medicine, St. Vincent's Hospital Clinical School, The University of New South Wales, Sydney, Australia; ${ }^{6}$ Department of Cancer Epidemiology, Cancer Institute, Chinese Academy of Medical Sciences, Peking Union Medical College, Beijing, China.
\end{abstract}

Email: "Surabhi_liyanage@hotmail.com

Received December $6^{\text {th }}$, 2012; revised January $7^{\text {th }}, 2013$; accepted February $9^{\text {th }}, 2013$

\begin{abstract}
Background: China has one of the highest incidence rates of oesophageal cancer in the world. The role of human papillomavirus (HPV) has been extensively researched in oesophageal squamous cell carcinoma (OSCC) with indeterminate results. The majority of these studies have been conducted in the Chinese population. Evidence for a definitive HPV-OSCC association could potentially support prophylactic vaccination in target populations, highlighting the need for ongoing investigation. The aim of this review is to summarise the findings of HPV DNA in OSCC tissue in Chinese subjects, with a view to informing further research in this area. Methods: A systematic literature search of the Chinese National Knowledge Infrastructure (CNKI) database, Medline, Embase and PubMed was conducted for all studies in English and Chinese language, examining OSCC tissue for HPV DNA in China. Reference lists of retrieved articles were reviewed and hand searches of relevant, key journals were conducted, to source articles which were not electronically indexed. Sixty-four studies met our selection criteria. Data from case-control and cross-sectional studies were analysed separately for any HPV-OSCC association, using the Epi Info ${ }^{\text {TM }} 3.5 .3$ software program. Results: From all studies conducted in the Chinese population, 2166/5953 (36\%) of all OSCC tissue and 478/1684 (28\%) of healthy control tissue, tested positive for HPV. We found that 11/16 case-control and cross-sectional studies had a statistically significant crude odds ratio, which supported a potential HPV-OSCC association. The largest study, carried out in the high incidence County of Anyang in Henan Province, reported 207/265 (78\%) OSCC tissues testing positive for HPV DNA against 203/357 (57\%) controls and had an unadjusted odds ratio of 2.71 (p-value < 0.0001). Conclusion: A rigorous meta-analysis would improve interpretation of the data and a well-designed large-scale case-control study is warranted. If a link is found between HPV and OSCC, prophylactic HPV vaccines could be of significant benefit in China.
\end{abstract}

Keywords: Human Papillomavirus; Oesophageal Carcinoma; Squamous Cell Carcinoma; HPV Vaccine; China

\section{Background}

The role of human papillomavirus (HPV) as a potential aetiological factor in oesophageal squamous cell carcinoma (OSCC) has been debated for the last three decades *HS currently holds a National Health and Medical Research Council (NHMRC) Training Fellowship (1012631)_Australian Based Public Health Fellowship). ATN currently holds a National Health and Medical Research Council (NHMRC) Training Fellowship (630724-Australian Based Public Health Fellowship). ATN has previously received research funding for other projects from a manufacturer of HPV vaccine.

${ }^{\#}$ Corresponding author.
[1]. The pathogenesis of HPV in cervical cancer is well established and the International Agency on Research on Cancer (IARC) has accepted the role of HPV in several head and neck cancers [2]. However, evidence for a definitive link between HPV and OSCC remains controversial.

The development of prophylactic HPV vaccines is predicted to have a major public health impact in the field of cervical cancer. If HPV is established as an aetiologic factor in OSCC, the prophylactic HPV vaccines may play an important part in reducing mortality from 
OSCC, particularly in a country such as China where oesophageal cancer (OC) contributes significantly to the nation's cancer burden.

\subsection{HPV}

Papillomaviruses have non-capsulated icosahedral virions which are approximately 55nm in diameter and contain a genome of approximately 8000 base pairs [3]. The genome is surrounded by 72 capsomeres. The outer coat of the virus is comprised of a major and minor capsid protein. The HPV genome is comprised of three major regions and consists of circular double-stranded DNA which codes for 8 proteins. The Early region (E1-8) codes for genes associated with transcription, plasmid replication and transformation. The Late region consists of genes which code for the major (L1) and minor (L2) capsid proteins. The control region is responsible for producing the vital factors in the regulation of transcription and replication [4].

HPV infections have been linked to a broad range of mucocutaneous diseases, from benign skin warts to premalignant lesions and invasive carcinoma. Of the currently characterized HPV types, infection has been described in epithelial layers of the skin, the anogenital region and the oropharyngeal mucosa [5].

\subsection{Human Papillomaviruses in Cancer}

Currently, it has been estimated that HPV is responsible for 5.1 percent of the global cancer burden [6,7]. The mechanism of oncogenesis of HPV in cervical cancer has been well documented and may also be applicable to oesophageal mucosa if HPV is an aetiologic factor in OSCC. The integration of viral DNA into the host genome appears to be an important step in establishing the pathway to carcinogenesis [8]. Integration of HPV disrupts the viral E2 gene, which acts as a negative regulator of the E6/E7, the main viral genes responsible for immortalization and malignant transformation of the infected host cell. With loss of E2 control, unregulated expression of the E6 and E7 oncoproteins cause proteolytic degradation of the p53 and retinoblastoma $(\mathrm{pRb})$ tumor suppressor genes respectively, effectively establishing malignancy $[8,9]$.

To date, there has been no definitive description of how HPV could infect the oesophagus. However, as the oesophageal mucosa is continuous with that of the oropharynx, hypotheses related to transmission in HPV related oropharyngeal cancers have also been extended to OCs. Consequently, higher numbers of sexual partners, increasing practice of oral sex and initiation of sexual encounters at an earlier age have been associated with HPV-related oropharyngeal malignancies and could simi- larly be one of the risk factors for OCs in which HPV is isolated [10-13].

Some reports have also suggested a transplacental mode of transmission of HPV from infected mothers to their babies in utero as well as during passage of the infant through the birth canal. [14] This is supported by findings of genital tract HPV types 6, 11, 16 and 18 (usually found in the genital tract), in oesophageal tissue of newborns [15].

\subsection{Oesophageal Squamous Cell Carcinoma}

Of the main histologic subtypes of OC, OSCC accounts for the majority of oesophageal malignancies worldwide and is the predominant form of OC diagnosed in African and Asian countries [16,17].

The main aetiologic factors for OSCC are discussed below. The onset and progress of oesophageal cancer is insidious with few early symptoms, resulting in advanced disease at time of diagnosis for many patients. Endoscopy and barium swallow are the mainstay of OSCC diagnosis, with follow up endosonography and chest and abdominal computer tomography scans used for staging [18]. Dysphagia, odynophagia, dyspnoea, significant weight loss and other symptoms and clinical signs related to disseminated disease are generally reported and observed in patients with advanced OSCC. Prognosis is often poor and the five-year survival rate in most cases, is less than $10 \%[18]$.

\subsection{Epidemiology of Oesophageal Cancer-How China Compares to the Rest of the Globe}

OC is the eighth most common malignancy worldwide with an incidence of an estimated 500,000 new cases annually [6]. Approximately half of the world's OC cases occur in China where a reported annual incidence of 250,000 cases makes OC the nation's second most common malignancy, after lung cancer [19,20]. OC has a poor prognosis as it is usually diagnosed late, with a five year survival of less than 5\% [21]. In 2008, oesophageal malignancy was responsible for 406,000 deaths globally, making it the sixth highest cause of cancer-related deaths [22] while in China, with an annual mortality of 150,000, it is the fourth leading cause of death from malignancy $[23,24]$. It is the second most common cause of cancer-related death in Chinese males [25]. Figure 1 depicts the age-standardized mortality rates per 100,000 for oesophageal cancer in China, from 1990-1992. The most recent survey on oesophageal cancer incidence and mortality in China was carried out from 1998 to 2002 (Table 1). From the 30 cities and counties included, Ci County in Hebei Province had the highest incidence and mortality rates in age-standardized calculations for both men 


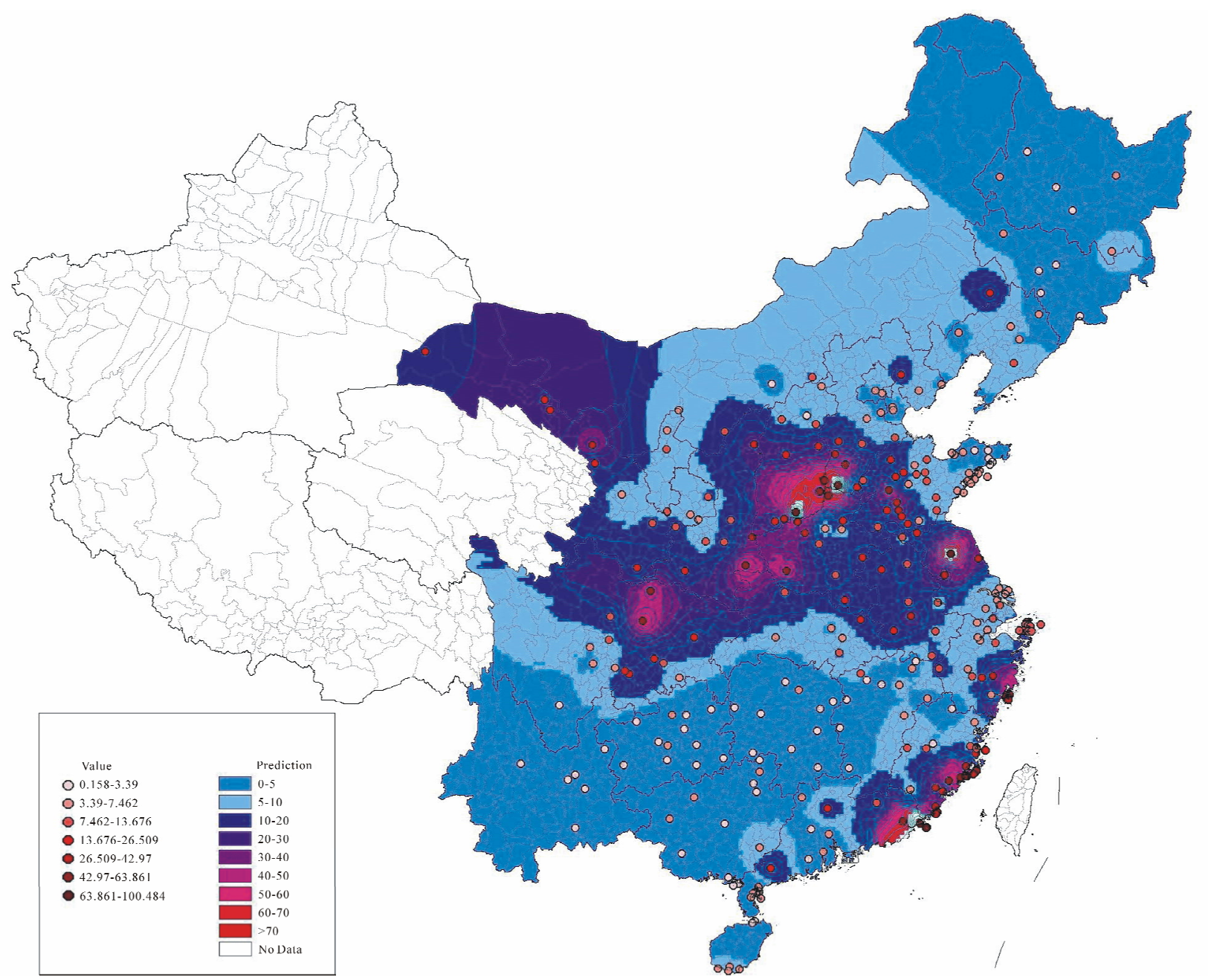

Figure 1. Estimated oesophageal cancer incidence per 100,000 in China, 1990-1992.

and women.

\subsection{Epidemiology of OSCC in China}

OSCC is predominantly a disease of developing nations and is the principal histologic type of OC in the Central Asian OC belt, which includes high-incidence countries such as China [26]. The average incidence rate for OC in the Chinese population is 13 per 100,000 [19] with OSCC representing more than $99 \%$ of all OC cases in China [27]. The variation in geographic incidence of OSCC internationally as well as within the same country, is well documented [28,29]. The major endemic regions within China are the northern Jiangsu province and the Linxian and Anyang counties in the eastern province of Henan $[19,30]$, with mortality rates as high as $161 / 100,000$ for males and 103/100,000 for females, in Linxian [31]. Significant differences in the incidence of OSCC also exist between regions of the same province in China, an intriguing and unexplained phenomenon. For instance, counties within the province of Hebei in the north of China, have reported incidence rates varying from 1.4 to 118.2 per 100,000 [32].

The broad range of incidence rates both regionally and globally has been ascribed to the complex, multifactorial aetiology of OSCC. In developed countries, tobacco use and excessive alcohol consumption are thought to be the two most important causative factors, responsible for $90 \%$ of OSCC cases [7,33-35]. However, in developing nations such as China, only a small percentage of OSCC cases can be attributed to alcohol and smoking [7,34,35]. In these high incidence areas, opium abuse, nutritional deficiencies [36,37], ingestion of hot food and beverages $[38,39]$, exposure to nitrosamines, industrial chemicals, and certain viruses [40-45] such as HPV have also been implicated.

\subsection{Evidence for Involvement of HPV in OSCC}

In 1982, the carcinogenic potential of HPV in OC was 
Table 1. Incidence and mortality rates for oesophageal cancer in China, 1998-2002 ${ }^{1}$ (data sourced from $\mathrm{Li} 2007$ ) [47].

\begin{tabular}{|c|c|c|c|c|c|}
\hline \multirow[t]{2}{*}{ CITY/COUNTY } & \multirow[t]{2}{*}{ REGION } & \multicolumn{2}{|c|}{ INCIDENCE (per 100,000) } & \multicolumn{2}{|c|}{ MORTALITY (per 100,000) } \\
\hline & & Male & Female & Male & Female \\
\hline Beijing & North & 5.8 & 2.5 & 4.2 & 1.9 \\
\hline Tianjin & North East & 5.5 & 2.2 & 4.6 & 1.9 \\
\hline Ci County, Hebei Province & North & 122.3 & 77.8 & 107.8 & 57.2 \\
\hline Sha County, Fujian Province & South East & 92.3 & 52.8 & 81.1 & 46.3 \\
\hline Yangcheng County, Shanxi Province & North East & 119.7 & 69.3 & 95.4 & 49.6 \\
\hline Dalian, Liaoning Province & North & 6.5 & 1.4 & 6.3 & 1.0 \\
\hline Anshan, Liaoning Province & North & 6.7 & 1.7 & 5.3 & 1.6 \\
\hline Harbin, Heilongjiang Province & North & 7.4 & 1.3 & 5.7 & 0.9 \\
\hline Shanghai & East & 6.5 & 2.1 & 5.6 & 1.7 \\
\hline Haimen, Jiangsu Province & East & 11.3 & 4.2 & 10.4 & 3.8 \\
\hline Qidong, Jiangsu Province & East & 6.3 & 2.5 & 5.8 & 2.1 \\
\hline Huaian, Jiangsu Province & East & 85.1 & 62.6 & 58.5 & 41.3 \\
\hline Yangzhong, Jiangsu Province & East & 67.7 & 62.5 & 57.5 & 47.5 \\
\hline Hangzhou, Zhejiang Province & East & 6.8 & 1.4 & 4.3 & 1.2 \\
\hline Jiaxing, Zhejiang Province & East & 9.9 & 3.0 & 10.1 & 2.2 \\
\hline Jiashan County, Zhejiang Province & East & 14.7 & 3.6 & 13.4 & 3.2 \\
\hline Haining, Zhejiang Province & East & 11.4 & 3.1 & 9.7 & 2.8 \\
\hline Changle, Fujian Province & South East & 12.9 & 4.1 & 11.0 & 3.5 \\
\hline Linqu County, Shandong Province & East & 16.5 & 3.1 & 15.1 & 3.3 \\
\hline Feicheng, Shandong Province & East & 61.7 & 27.8 & 55.1 & 25.3 \\
\hline Linzhou, Henan Province & East & 74.5 & 51.8 & 63.7 & 37.2 \\
\hline Wuhan, Hubei Province & Central & 8.6 & 2.7 & 7.1 & 2.1 \\
\hline Guangzhou, Guangdong Province & South East & 7.0 & 1.4 & 6.0 & 0.9 \\
\hline Shenzhen, Guangdong Province & South East & 38.7 & 21.1 & 6.6 & 3.1 \\
\hline Sihui, Guangdong Province & South East & 6.0 & 1.7 & 6.7 & 1.8 \\
\hline Zhongshan, Guangdong Province & South East & 12.6 & 1.4 & 9.5 & 1.0 \\
\hline Fusui County, Guangxi Province & South & 3.3 & 0.7 & 3.6 & 0.6 \\
\hline Yanting County, Sichuan Province & West & 99.5 & 58.9 & 83.4 & 48.7 \\
\hline Gejiu, Yunnan Province & South & 0.5 & 0.0 & NS & NS \\
\hline Wuwei, Gansu Province & North West & 53.4 & 21.8 & NS & NS \\
\hline
\end{tabular}

NS-not specified.

first postulated by Syrjänen following the observation of HPV-related histological changes in OSCC tissue samples, identical to those seen in condylomas [46]. Subse-

${ }^{1}$ Rates are all age-standardized by Chinese standard population. quent investigations to assess a possible HPV-OSCC link, including experiments in animal models, serological, in vitro, and morphologic studies, have been well-documented $[1,29]$. Hypothesised modes of transmission of 
HPV in OSCC and mechanisms of oncogenesis based on a cervical cancer model, have also been previously summarized [28].

The most convincing studies have demonstrated the presence of HPV DNA sequences in OSCC tissue using techniques varying from Southern Blot to polymerase chain reaction (PCR), in situ hybridization (ISH) and immunohistochemistry (IHC). To date, the largest number of studies investigating the role of HPV in OSCC have been carried out in China, with some of these studies being published only in the Chinese language literature. To the best of our knowledge there have been no previous reports on this topic, which assess papers from both the English and Chinese language. We aim to review all studies conducted in China, in English and Chinese, with a view to informing prevention of OSCC in China through the use of prophylactic HPV vaccines, should an aetiological link to HPV be confirmed.

\section{METHODS}

\subsection{Search Strategy}

English and Chinese language papers included in this review were identified by searching the CNKI database as well as Medline, Embase and PubMed. Search terms included "human papillomavirus", "HPV”, "oesophageal cancer", "squamous cell carcinoma" and "China". In addition, reference lists of retrieved articles were reviewed and hand searches of key journals including Annals of Oncology, Lancet Oncology, Anticancer Research, Gastroenterology, International Journal of Cancer, BMC Cancer, Diseases of the Esophagus, World Journal of Gastroenterology, Cancer Epidemiology Biomarkers \& Prevention and Journal of Clinical Pathology, were conducted to source any articles which were not electronically indexed. There were no limitations to date of publication for either English or Chinese language studies and papers were sourced from the date when the databases started until February 2012.

\subsection{Data Extraction}

Articles met the selection criteria if they investigated the presence of HPV DNA in OSCC tissue in a Chinese cohort. All study types which included case series, crosssectional and case-control studies, were accepted. Papers were searched and data were extracted by one author (SSL). All studies which met our search criteria were tabulated in chronological order (Table 2).

For each paper, data extraction included: 1) the year in which the study was conducted; 2) the geographic region of China from which subjects were recruited (Tables 2 and 3, Figure 2); 3) the testing methodology; 4) HPV types detected; 5) number of HPV positive OSCC sam- ples compared to total number of OSCC samples tested; 6) if applicable, number of HPV positive controls compared to total number of control specimens tested; 7) the type of study; and 8) specimen retrieval method.

Recording of the specimen retrieval method is intended to assess whether HPV detection rates differ between deep and superficial OSCC test specimens, the sample retrieval method was recorded for all studies. Deep tissue was classified as surgical resections, diagnostic biopsies and formalin fixed and paraffin embedded samples; while superficial specimens included cell brushings and balloon cytology samples (Table 2).

The Chinese literature was also searched for the most recent epidemiological data on oesophageal cancer incidence and mortality and a summary of the results obtained from the source [47] are presented in Table 1. In addition, authors of this review, based at the Beijing Cancer Institute \& Hospital Chinese Academy of Medical Sciences (CICAMS), generated a map (Figure 1) of oesophageal cancer mortality using data collected by CICAMS from 1990-1992, on 10\% of the Chinese population. Based on OC mortality data collected for various counties in China, predicted mortality rates have been projected for surrounding regions (Figure 1).

\subsection{Analysis of Case-Control and Cross-Sectional Studies}

The case-control study design allows the investigator to estimate the odds of an outcome, such as OSCC, occurring when exposure to a potential risk factor such as HPV, has taken place. It is particularly useful as an initial study to determine causality, if a link between the exposure and outcome of interest, has not been previously established [48]. The case-control methodology is both time and cost-effective when investigating diseases with long latency periods, such as OSCC, because the disease state already exists at the start of the investigation [48]. Furthermore, case-control study design allows the simultaneous assessment of multiple risk factors, which is useful in diseases such as OSCC, which have a multifactorial aetiology [49]. Thus case-control studies are the most practical study design for examining the research question of whether HPV is an aetiological factor in OSCC.

This review defines cases as patients with OSCC and controls as healthy subjects from whom macroscopically normal oesophageal biopsy samples have been obtained. Papers which identify paraoesophageal tissue from OC patients, as controls, were not acknowledged in the control column of Table 2 and were not classified as casecontrol studies in Table 4, as there is a significant possibility of cross-contamination and spread of HPV from the tumour into adjacent tissue, resulting in false-positive 
Table 2. Identified studies of HPV in OSCC in China in English and Chinese language literature ${ }^{2}$.

\begin{tabular}{|c|c|c|c|c|c|c|c|c|c|}
\hline $\begin{array}{c}\text { Year of } \\
\text { publication }\end{array}$ & $\begin{array}{c}\text { City/ } \\
\text { province }\end{array}$ & $\begin{array}{l}\text { Region of } \\
\text { china }\end{array}$ & $\begin{array}{l}\text { Detection } \\
\text { method }\end{array}$ & $\begin{array}{l}\text { HPV types } \\
\text { detected }\end{array}$ & $\begin{array}{l}\text { Method of oscc } \\
\text { specimen retrieval }\end{array}$ & $\begin{array}{l}\text { No. of hpv positive } \\
\text { oscc samples/total } \\
\text { tested (\% hpv } \\
\text { detection) }\end{array}$ & $\begin{array}{l}\text { No. of positive } \\
\text { control } \\
\text { samples/total } \\
\text { tested (\% hpv } \\
\text { detection) }\end{array}$ & $\begin{array}{l}\text { Type of } \\
\text { study }\end{array}$ & Ref \\
\hline 1989 & henan & east & ihc & ag & ns & 6/31 (19) & $\mathrm{n} / \mathrm{a}$ & case series & {$[51]$} \\
\hline 1990 & linxian, henan & east & $\mathrm{hb}$ & - & surgical specimen & 25/51 (49) & $\mathrm{n} / \mathrm{a}$ & case series & [102] \\
\hline 1990 & linxian, henan & east & fish & $11,16,18$ & surgical specimen & $53 / 80(66)$ & $\mathrm{n} / \mathrm{a}$ & case series & [102] \\
\hline 1990 & linxian, henan & east & ish & $6,11,16,18$ & surgical specimen & 22/51 (43) & $\mathrm{n} / \mathrm{a}$ & case series & [102] \\
\hline $1991^{3}$ & chengdu, sichuan & south west & $\mathrm{sb}$ & 16 & surgical specimen & $12 / 24(50)$ & $\mathrm{n} / \mathrm{a}$ & case series & [103] \\
\hline 1992 & linxian, henan & east & pcr & $6,11,16,18$ & surgical specimen & 25/51 (49) & $\mathrm{n} / \mathrm{a}$ & case series & {$[15]$} \\
\hline 1992 & linxian, henan & east & sb & $11,16,18,30$ & surgical specimen & $8 / 20(40)$ & $\mathrm{n} / \mathrm{a}$ & case series & [15] \\
\hline $1993^{3}$ & fujian & south east & pcr & ср & ffpe & $24 / 40(60)$ & $\mathrm{n} / \mathrm{a}$ & case series & {$[56]$} \\
\hline 1993 & linxian, henan & east & ish & $\begin{array}{c}6,11,16 \\
18,30\end{array}$ & surgical specimen & 85/363 (23) & $\mathrm{n} / \mathrm{a}$ & case series & [108] \\
\hline 1994 & fuzhou, fujian & south east & pcr & 6,16 & surgical specimen & $24 / 40(60)$ & $\mathrm{n} / \mathrm{a}$ & case series & {$[57]$} \\
\hline $1995^{3}$ & linxian, henan & east & sb, pcr & 16,18 & surgical specimen & 0/35 (0) & $\mathrm{n} / \mathrm{a}$ & case series & {$[53]$} \\
\hline $1996^{c}$ & zhengzhou, henan & east & ish & $6,11,16,18$ & ffpe & $22 / 40$ (55) & $\mathrm{n} / \mathrm{a}$ & case series & [104] \\
\hline 1996 & beijing & north & pcr & $6,16,18$ & surgical specimen & $3 / 70(4)$ & $\mathrm{n} / \mathrm{a}$ & case series & {$[77]$} \\
\hline $1996^{c}$ & sichuan & south west & sb, pcr & 16,18 & ffpe & 37/103 (36) & $\mathrm{n} / \mathrm{a}$ & case series & {$[60]$} \\
\hline 1997 & sichuan & south west & pcr & 16,18 & surgical specimen & 32/152 (21) & $\mathrm{n} / \mathrm{a}$ & case series & {$[25]$} \\
\hline 1997 & ns & north & ish & $\begin{array}{l}\text { wide } \\
\text { spectrum }\end{array}$ & surgical specimen & 3/36 (8) & $\mathrm{n} / \mathrm{a}$ & case series & [105] \\
\hline 1999 & anyang, henan & east & pcr & ср & $\begin{array}{l}\text { diagnostic biopsies } \\
(3 / 70) \text {, surgical } \\
\text { specimens }(7 / 23) \text {, } \\
\text { scrapings }(10 / 24)\end{array}$ & 20/117 (17) & $\mathrm{n} / \mathrm{a}$ & case series & {$[59]$} \\
\hline 1999 & ns & ns & pcr & $\begin{array}{c}6,9,18,20 \\
24,51,52,57\end{array}$ & surgical specimen & 10/29 (34) & $\mathrm{n} / \mathrm{a}$ & case series & {$[62]$} \\
\hline $2000^{3}$ & $\begin{array}{l}\text { shaanxi \& gansu } \\
\text { \& sichuan \& } \\
\text { shanxi \& } \\
\text { hunan }\end{array}$ & $\begin{array}{c}\text { central \& } \\
\text { north west \& } \\
\text { west \& north } \\
\quad \text { east }\end{array}$ & pcr & 11,16 & surgical specimen & ns/22 (ns) & $\mathrm{n} / \mathrm{a}$ & case series & [95] \\
\hline 2000 & linxian, henan & east & pcr & ср & surgical specimen & 18/101 (17) & $\mathrm{n} / \mathrm{a}$ & case series & [123] \\
\hline 2000 & linxian, henan & east & ish & $\begin{array}{l}6,11,16 \\
18,30,53\end{array}$ & surgical specimen & $117 / 700(17)$ & $\mathrm{n} / \mathrm{a}$ & case series & {$[82]$} \\
\hline $2000^{3}$ & shaanxi & central & ihc & e6 & ffpe & $44 / 60(73)$ & $24 / 56(43)$ & case control & [101] \\
\hline 2000 & beijing & north & pcr & ср & surgical specimen & ns/37 (ns) & $\mathrm{n} / \mathrm{a}$ & case series & [94] \\
\hline $2001^{3}$ & anyang, henan & east & is-pcr, ish & 11, e6, e7 & surgical specimen & $18 / 30(60)$ & $\mathrm{n} / \mathrm{a}$ & case series & {$[71]$} \\
\hline 2001 & $\begin{array}{l}\text { shangzhuang- } \\
\text { anyang \& } \\
\text { tangmiao- } \\
\text { neihuang }\end{array}$ & east & pcr, ish & 16 & ball cytology & 2/2 (100) & 50/112 (44) & $\begin{array}{c}\text { cross } \\
\text { sectional }\end{array}$ & {$[65]$} \\
\hline 2001 & linxian, henan & east & pcr & ср & ball cytology & 2/32 (6) & 4/57 (7) & case control & {$[72]$} \\
\hline 2002 & anyang, henan & east & pcr, ish & 16,18 & ball cytology & 39/62 (63) & 17/36 (47) & case control & {$[64]$} \\
\hline 2002 & eastern guandong & south east & pcr & ср & surgical specimen & 115/176 (66) & $\mathrm{n} / \mathrm{a}$ & case series & [73] \\
\hline
\end{tabular}




\begin{tabular}{|c|c|c|c|c|c|c|c|c|c|}
\hline $2003^{3}$ & ns & ns & pcr & ср & surgical specimen & $28 / 40(70)$ & $\mathrm{n} / \mathrm{a}$ & case series & {$[78]$} \\
\hline $2003^{3}$ & cixian, hebei & north & pcr, ihc & ср & surgical specimen & 28/152 (18) & $\mathrm{n} / \mathrm{a}$ & case series & [68] \\
\hline 2003 & anyang \& shanxi & $\begin{array}{c}\text { east \& north } \\
\text { east }\end{array}$ & pcr,ihc,ish & 16 & surgical specimen & $31 / 48$ (65) & $\mathrm{n} / \mathrm{a}$ & case series & [80] \\
\hline 2003 & $\begin{array}{l}\text { hong kong \& } \\
\text { sichuan \& } \\
\text { linxian \& } \\
\text { shantou \& } \\
\text { xi'an }\end{array}$ & $\begin{array}{c}\text { south \& } \\
\text { south west \& } \\
\text { east \& south } \\
\text { east \& central }\end{array}$ & pcr & 16,18 & surgical specimen & 43/319 (13) & $\mathrm{n} / \mathrm{a}$ & case series & {$[76]$} \\
\hline 2004 & $\begin{array}{c}\text { hong kong \& } \\
\text { sichuan \& linxian }\end{array}$ & $\begin{array}{l}\text { south \& west } \\
\text { \& east }\end{array}$ & pcr & 16,18 & surgical specimen & 18/87 (21) & $\mathrm{n} / \mathrm{a}$ & case series & [75] \\
\hline 2004 & xinjiang & north west & pcr & 16 & surgical specimen & 55/104 (53) & $\mathrm{n} / \mathrm{a}$ & case series & [69] \\
\hline $2004^{3}$ & beijing & north & ihc & e6, e7 & ffpe & $\begin{array}{c}15 / 18,16 / 18 \\
(83,89)\end{array}$ & $\mathrm{n} / \mathrm{a}$ & case series & [100] \\
\hline $2004^{3}$ & guandong & south east & ihc, pcr & 16 & ffpe & $14 / 30(47)$ & $7 / 60(12)$ & case control & [83] \\
\hline $2005^{3}$ & anyang \& beijing & east \& north & ish & 16 & ffpe & $86 / 119(72)$ & $\mathrm{n} / \mathrm{a}$ & case series & [106] \\
\hline 2005 & anyang, henan & east & pcr & 16,18 & surgical specimen & 207/265 (78) & 203/357 (57) & case control & [55] \\
\hline $2005^{3}$ & jiangsu & east & pcr, hb & ns & cell brushing & 23/60 (38) & $11 / 60(18)$ & case control & [85] \\
\hline 2006 & shantou & south east & ish & 16,18 & surgical specimen & $24 / 60(40)$ & $\mathrm{n} / \mathrm{a}$ & case series & [111] \\
\hline 2006 & henan \& hubei & east \& central & ish, ihc & 16,18 & surgical specimen & ns/82 (ns) & ns & unk & [107] \\
\hline 2006 & linxian, henan & east & ish & - & ball cytology & $0 / 4(0)$ & $61 / 475$ (13) & $\begin{array}{c}\text { cross } \\
\text { sectional }\end{array}$ & {$[52]$} \\
\hline $2007^{3}$ & shanghai & east & ish & 16 & surgical specimen & $59 / 90$ & $2 / 20$ & case control & [110] \\
\hline $2007^{3}$ & xinjiang & north west & pcr & $18,31,45$ & ffpe & ns/316 (ns) & $\mathrm{n} / \mathrm{a}$ & case series & {$[96]$} \\
\hline $2007^{3}$ & sichuan & south west & pcr & 16 & surgical specimen & 43/112 (38) & $\mathrm{n} / \mathrm{a}$ & case series & {$[67]$} \\
\hline 2007 & anyang, henan & east & pcr & $\begin{array}{l}16,31,51 \\
56,53,73\end{array}$ & surgical specimen & $11 / 100(11)$ & $\mathrm{n} / \mathrm{a}$ & case series & [58] \\
\hline 2007 & $\begin{array}{l}\text { gansu \& } \\
\text { shandong }\end{array}$ & $\begin{array}{c}\text { north west \& } \\
\text { east }\end{array}$ & pcr, sb & 16,18 & surgical specimen & 19/59 (32) & $\mathrm{n} / \mathrm{a}$ & case series & [74] \\
\hline $2007^{3}$ & $\begin{array}{c}\text { anyang \& } \\
\text { zhengzhou, henan }\end{array}$ & east & pcr, hb & 16 & surgical specimen & $54 / 110$ (49) & $7 / 45$ (16) & case control & [87] \\
\hline $2008^{3}$ & linzhou, henan & east & pcr & 16,18 & surgical specimen & 29/31 (94) & $\mathrm{n} / \mathrm{a}$ & case series & {$[63]$} \\
\hline 2008 & xinjiang & north west & pcr & $\begin{array}{c}6,11,16,18, \\
31,52,66\end{array}$ & surgical specimen & 20/67 (30) & $\mathrm{n} / \mathrm{a}$ & case series & {$[70]$} \\
\hline $2008^{3}$ & xinjiang & north west & pcr & 16 & ffpe & $58 / 150$ (39) & $4 / 40(10)$ & case control & {$[84]$} \\
\hline $2008^{3}$ & chongqing & central & pcr & 16,18 & surgical specimen & 43/112 (38) & $\mathrm{n} / \mathrm{a}$ & case series & {$[88]$} \\
\hline $2009^{3}$ & $\begin{array}{c}\text { guandong \& } \\
\text { henan }\end{array}$ & $\begin{array}{c}\text { south east \& } \\
\text { east }\end{array}$ & pcr & $\begin{array}{l}16,18,45, \\
33,58,59, \\
73,31,56\end{array}$ & surgical specimen & $0 / 140(0)$ & $\mathrm{n} / \mathrm{a}$ & case series & [54] \\
\hline $2009^{3}$ & henan & east & pcr & 16 & surgical specimen & $37 / 44(84)$ & $\mathrm{n} / \mathrm{a}$ & case series & {$[97]$} \\
\hline $2009^{3}$ & hebei & north & pcr & 16,18 & surgical specimen & $37 / 42(88)$ & $\mathrm{n} / \mathrm{a}$ & case series & [79] \\
\hline $2009^{3}$ & xinjiang & north west & pcr & 16 & ffpe & 23/63 (37) & 21/126 (17) & case control & {$[66]$} \\
\hline $2009^{3}$ & xinjiang & north west & pcr & 16 , e6 & surgical specimen & 26/82 (32) & $\mathrm{n} / \mathrm{a}$ & case series & {$[91]$} \\
\hline 2010 & shantou & south east & pcr & $16,18,58$ & surgical specimen & $35 / 70(50)$ & 20/60 (33) & case control & [99] \\
\hline
\end{tabular}




\section{Continued}

\begin{tabular}{|c|c|c|c|c|c|c|c|c|c|}
\hline 2010 & henan & east & pcr & 16 & surgical specimen & 8/17 (47) & $\mathrm{n} / \mathrm{a}$ & case series & [98] \\
\hline 2010 & $\begin{array}{c}\text { xinjiang \& } \\
\text { anyang \& } \\
\text { shantou }\end{array}$ & $\begin{array}{l}\text { north west \& } \\
\text { east \& south } \\
\text { east }\end{array}$ & pcr & $\begin{array}{l}6,16,18,26 \\
45,56,57,58\end{array}$ & ns & $160 / 347$ (55) & $\mathrm{n} / \mathrm{a}$ & case series & [124] \\
\hline 2010 & linxian, henan & east & pcr & $89,16,31$ & surgical specimen & 3/267 (1) & $\mathrm{n} / \mathrm{a}$ & case series & [61] \\
\hline 2010 & shaanxi & central & pcr & 16 & surgical specimen & 35/69 (51) & 2/32 (6) & case control & [89] \\
\hline $2010^{c}$ & xinjiang & north west & pcr & 16 , e6 & ffpe (biopsy) & 46/100 (46) & $22 / 100(22)$ & case control & [86] \\
\hline 2011 & changhua, taiwan & south east & pcr, ish & $6,11,16,18$ & surgical specimen & 2/31 (6) & $\mathrm{n} / \mathrm{a}$ & case series & {$[93]$} \\
\hline $2011^{\mathrm{c}}$ & jiangsu & east & ish & 16,18 & ffpe & 40/72 (56) & 7/48 (15) & case control & [109] \\
\hline $2011^{\mathrm{c}}$ & linzhou, henan & east & pcr & 16 & diagnostic biopsy & 18/18 (100) & $\mathrm{n} / \mathrm{a}$ & case series & [92] \\
\hline
\end{tabular}

Ag-HPV Antigens; CP_consensus primers; GP_-general primers; HB-histological biopsy; IHC-immunohistochemistry; ISH-in situ hybridization; PCR - Polymerase chain reaction; SB-southern blot hybridization; FFPE—formalin fixed and paraffin embedded; N/A — Not applicable as study did not include controls; ND—Not Determined; UNK—Unknown due to insufficient information for determining study type; NS—not specified.

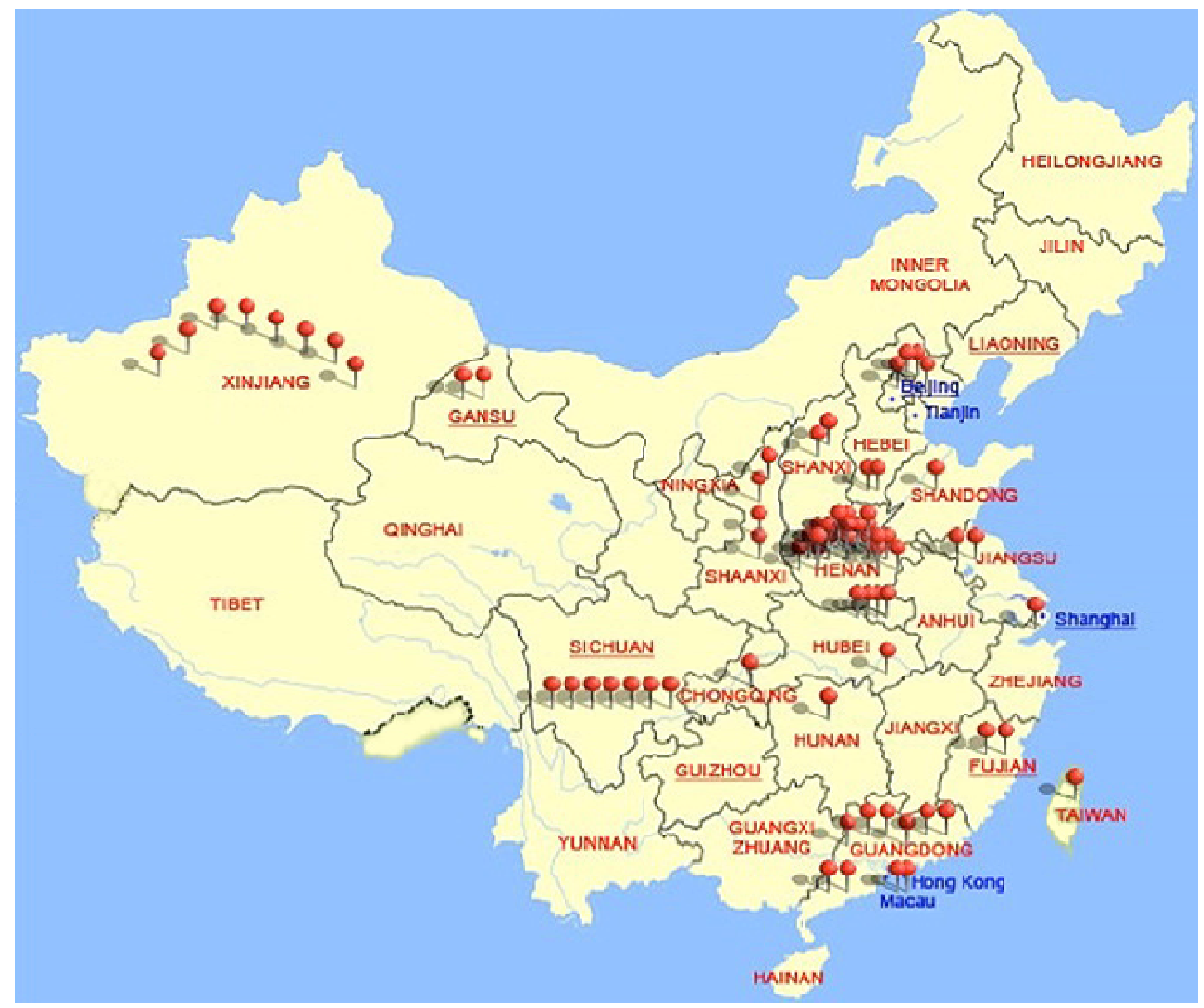

Figure 2. Representation of the number of HPV-OSCC studies carried out in various provinces in China.

HPV detection rates. We identified 14 case control studies and a further 2 cross-sectional studies (Table 4) in- vestigating OSCC tissue compared to oesophageal tissue from healthy controls, for the presence of HPV DNA in

${ }^{2}$ Control subjects in this paper are defined as individuals who do not have a diagnosis of OSCC. Therefore studies which have determined controls to be biopsies of macroscopically normal oesophageal tissue adjacent to the primary tumour, have been discounted;

${ }^{3}$ Chinese language papers. The remaining papers are in English. 
Table 3. HPV Detection Rate by Region in China, based on 64 identified studies from the English and Chinese Literature ${ }^{\mathrm{d}}$.

\begin{tabular}{cc}
\hline REGION & $\begin{array}{c}\text { NO OF OSCC POSITIVE SAMPLES/TOTAL } \\
\text { TESTED (\% HPV DETECTION) }\end{array}$ \\
North & $154 / 447(34)$ \\
North West & $228 / 566(40)$ \\
South East & $300 / 643(47)$ \\
South West & $124 / 391(32)$ \\
South & $16 / 123(13)$ \\
East & $1173 / 3415(34)$ \\
Central & $132 / 298(44)$ \\
Unspecified & $38 / 69(55)$ \\
Regions & \\
China (Total) & $2165 / 5952(36)$ \\
\hline
\end{tabular}

the Chinese population. Epi Info ${ }^{\mathrm{TM}} 3.5 .3$ [50] was used to calculate odds ratios (OR) with $95 \%$ confidence intervals, for the association of HPV with OSCC, by cross-tabulating the summary data presented in the papers for case-control and cross-sectional studies. P-values for the significance of the ORs were calculated from chi-squared test. Only two of the identified studies presented calculations of ORs. Few studies adjusted for confounding factors and our calculation of unadjusted odds ratios for the association of HPV with OSCC from the summary data provided in the papers, must therefore be interpreted in this context.

\section{Results}

The first study looking for an aetiological link between HPV and OSCC in China was carried out in 1989 using immunohistochemistry (IHC) [51]. In total, 64 studies

Table 4. Case-Control and Cross-Sectional Studies Examining HPV DNA in OSCC in China, from English and Chinese language literature ${ }^{4}$.

\begin{tabular}{|c|c|c|c|c|c|c|c|c|}
\hline YEAR & REGION & METHOD & $\begin{array}{l}\text { HPV } \\
\text { TYPES } \\
\text { FOUND }\end{array}$ & $\begin{array}{l}\text { POSITIVE NUMBER } \\
\text { CASES (\%) }\end{array}$ & $\begin{array}{c}\text { POSITIVE NUMBER } \\
\text { CONTROLS (\%) }\end{array}$ & $\begin{array}{l}\text { CRUDE ODDS } \\
\text { RATIO }^{5}\end{array}$ & P-VALUE & REF \\
\hline 2000 & Shaanxi (Central) & IHC & E6 & $44 / 60(73)$ & $24 / 56(43)$ & $3.67(1.57-8.65)$ & 0.0009 & {$[101]$} \\
\hline 2001 & $\begin{array}{l}\text { Shangzhuang - Anyang } \\
\text { \& Tangmiao - Neihuang } \\
\text { (East) }\end{array}$ & PCR, ISH & 16 & $2 / 2(100)$ & $50 / 112(44)$ & Incalculable & 0.1192 & [65] \\
\hline 2001 & Linxian, Henan (East) & PCR & $\mathrm{CP}$ & 2/32 (6) & $4 / 57(7)$ & $0.88(0.1-6.13)$ & 0.8898 & [72] \\
\hline 2002 & Anyang, Henan (East) & PCR, ISH & 16,18 & 39/62 (63) & $17 / 36(47)$ & $1.90(0.76-4.75)$ & 0.1305 & [64] \\
\hline 2004 & Guandong (SE) & IHC, PCR & 16 & $14 / 30(47)$ & 7/60 (12) & $6.63(2.04-22.23)$ & 0.0002 & [83] \\
\hline 2005 & Anyang, Henan (East) & PCR & 16,18 & 207/265 (78) & 203/357 (57) & $2.71(1.86-3.94)$ & $<0.0001$ & [55] \\
\hline 2005 & Jiangsu (East) & PCR, HB & NS & $23 / 60(38)$ & $11 / 60(18)$ & $2.77(1.12-6.97)$ & 0.0151 & [85] \\
\hline 2006 & Linxian, Henan (East) & ISH & Nil & $0 / 4(0)$ & $61 / 475(13)$ & $0.00(0-10.68)$ & 0.4429 & [52] \\
\hline 2007 & $\begin{array}{c}\text { Anyang \& } \\
\text { Zhengzhou, Henan } \\
\text { (East) }\end{array}$ & PCR, HB & 16 & $54 / 110(49)$ & $7 / 45$ (16) & $5.23(2.02-14.12)$ & 0.0001 & [87] \\
\hline 2007 & Shanghai (East) & ISH & 16 & $59 / 90(66)$ & $2 / 20(10)$ & $17.13(3.46$ - 114.6) & $<0.0001$ & {$[110]$} \\
\hline 2008 & Xinjiang (NW) & PCR & 16 & $58 / 150(39)$ & $4 / 40(10)$ & $5.67(1.8-19.87)$ & 0.0006 & [84] \\
\hline $2009^{6}$ & Xinjiang (NW) & PCR & 16 & 23/63 (37) & 21/126 (17) & $2.88(1.36-6.11)$ & 0.0023 & [66] \\
\hline 2010 & Shaanxi (Central) & PCR & 16 & $35 / 69$ (51) & $2 / 32(6)$ & $15.44(3.20-101.46)$ & $<0.0001$ & [89] \\
\hline 2010 & Shantou (SE) & PCR & $16,18,58$ & $35 / 70(50)$ & 20/60 (33) & $2.00(0.92-4.35)$ & 0.0552 & [99] \\
\hline $2010^{7}$ & Xinjiang (NW) & PCR & 16, E6 & $46 / 100(46)$ & $22 / 100(22)$ & $3.02(1.56-5.86)$ & 0.0003 & [86] \\
\hline 2011 & Jiangsu (East) & ISH & 16,18 & $40 / 72(56)$ & 7/48 (15) & $7.32(2.69-20.71)$ & $<0.0001$ & {$[109]$} \\
\hline \multicolumn{4}{|c|}{ Total } & $681 / 1239(55)$ & 478/1684(28) & & & \\
\hline
\end{tabular}

CP—consensus primers; HCII—Hybrid Capture 2; IHC—immunohistochemistry; ISH—in situ hybridization; LR—Low-risk HPV types; HR—High risk HPV types; PCR-Polymerase chain reaction.

\footnotetext{
${ }^{4}$ Table excludes studies which did not report the number of HPV positive OSCC samples from the total tested.

${ }^{5}$ Unadjusted ORs calculated using Epi Info ${ }^{\text {тM }}$ 3.5.3, from summary data presented in the papers. As adjustments for confounding factors have not been carried out in most studies, it is important to interpret these ORs with caution. Only two studies calculated ORs as highlighted below.

${ }^{6}$ OR calculated by authors of study: 2.67 (1.38 - 5.17); $\mathrm{P}<0.05$.

${ }^{7}$ OR calculated by authors of study: $3.020 ; \mathrm{P}<0.001$.
} 
have been conducted in China to date, which include a total of 6409 OSCC samples as summarised in Table 2. Of these, 36 were in the English language and 29 were in Chinese. Of the 64 studies investigating the role of HPV in OSCC in China, a majority of 47 were case series, with 14 case-control studies, 2 cross-sectional studies and 1 report of indeterminable study design (Table 2). From all studies conducted in the Chinese population, 2166/5953 (36\%) of all OSCC tissue and 478/1684 (28\%) of all healthy control tissue, tested positive for HPV (Table 2).

\subsection{Regions of China}

To date, the Chinese population has contributed the largest number of OSCC specimens for HPV analysis, compared to any other country [28]. Henan province in East China is the site of over $50 \%$ of all national studies (Figure 2). Of all OSCC samples tested in this high incidence area, 34\% yielded positive HPV results (Table 3). Other high-incidence areas within China from the north-west, south-east, and the central regions have reported even higher rates of HPV DNA detection in OSCC tissue, ranging from $40 \%$ - 47\% (Table 3 ).

\subsection{Testing Methods}

As summarized in Table 2, a variety of techniques have been used in these studies to detect HPV. The detection rate of HPV in OSCC tissue varied from 0 to $100 \%$. Only three studies did not detect HPV in any of the OSCC samples tested [52-54]. Forty-nine of 64 studies utilized PCR with HPV detection rates varying widely from $0 \%$ 100\% [15,25,53-99]. PCR yielded a HPV positive rate in $37 \%$ of all OSCC tissue tested using this method. Approximately one quarter of all OSCC specimens were analysed by ISH, with HPV detected in $30 \%$ of these samples. Six studies have used IHC to test OSCC tissues samples with HPV detection rates varying from $18 \%$ $89 \%[51,68,80,83,100,101]$. One study reported a $66 \%$ HPV detection rate using filter in situ hybridisation (FISH) [102], five studies used southern blot hybridization [15, $53,60,74,103]$ and a further three studies examined histological biopsies of oesophageal lesions $[85,87,102]$.

Sixteen studies used ISH with percentage of OSCC tissues testing positive for HPV, varying from $0 \%-72 \%$ $[52,64,65,71,80,82,93,102,104-111]$. ISH was the methodology used in the largest study to be carried out on this topic, analyzing a total of 700 OSCC samples from the Henan Province, with an HPV detection rate of $17 \%$ [82]. High-risk HPV types 16 and 18 were the most commonly detected genotypes in this study, as well as in all other investigations from the Chinese cohort.

\subsection{Site of Specimen Retrieval}

We also examined studies for any potential relationship between HPV detection rates and whether OSCC test specimens were superficial cell scrapings or deep tissue biopsies. Of the 60 studies which had sufficient information for analysis, one tested both superficial and deep oesophageal tissue specimens, 54 tested only deep tissue and the remaining 5 tested superficial specimens (Table 2). We found that 76/184 (41\%) of superficial OSCC samples and 1990/5607 (35\%) of deep OSCC tissue samples tested positive for HPV DNA.

\subsection{Results of Case-Control and Cross-Sectional Studies}

As highlighted in Table 4, we identified 14 case-control studies and 2 cross-sectional studies which investigate the association of HPV DNA in OSCC tissue compared to normal control tissue in the Chinese population. From these studies, 681/1239 (55\%) of all OSCC samples and 478/1684 (28\%) of all healthy control tissue tested positive for HPV. Only two of these studies calculated an odds ratio in the published papers $[66,86]$. Table 4 presents these results as well as our unadjusted, crude odds ratios which were calculated using Epi Info ${ }^{\mathrm{TM}}$ 3.5.3 (CDC) [50]. Our analysis demonstrates that $11 / 16$ studies had statistically significant odds ratios which support a potential HPV-OSCC link. The largest study, carried out in the high incidence County of Anyang in Henan Province [55], reported 207/265 (78\%) OSCC tissues testing positive for HPV DNA against 203/357 (57\%) controls and had an unadjusted odds ratio of 2.71 (p-value $<0.0001$ ).

\section{Discussion}

Our findings of a statistically significant association of HPV with OSCC in 11/16 case-control and cross-sectional Chinese studies, suggest that HPV may be a potential aetiological factor in OSCC, in the Chinese population. However, as our unadjusted ORs have been calculated from summary data in papers, many of which have not adjusted for important confounding factors, it is important to interpret our results accordingly.

In the 16 studies in China which have tested control subjects for HPV [52,55,64-66,72,83-87,89,99,101,109, 110], a number of controls have tested positive for HPV, including two studies in which HPV was isolated from a greater percentage of controls than cases [52,72]. Interestingly, both of these studies were carried out in Linxian within Henan province, a region which has one of the highest incidence rates of OC in the world [30]. Fidalgo et al. also reported $100 \%$ of controls testing positive for HPV DNA in a Portuguese cohort [112]. This trend may be suggestive of an early role for HPV in the aetiology of 
OSCC, in which normal oesophageal mucosa infected by HPV may undergo malignant transformation following expression of the E6/E7 viral oncogenes [77].

We found that HPV types 16 and 18 are the most commonly detected genotypes within oesophageal tissue in China. However, it is also important to note that a majority of studies to date test only for the main oncogenic genotypes of HPV, namely types 16 and 18, thereby raising the possibility that less common HPV types are missed in the testing process. Nevertheless, some HPV types, which have not previously been isolated from oesophageal tissue, have been described in some studies which have tested for a broader range of HPV types. Of note are types 30, 53, 56, 66, 73 and 89 in the Chinese cohort (Table 2). In particular, Chang et al., reported 8 out of $85 \mathrm{HPV}$-positive OSCC samples with HPV 30 [108]. As HPV 30 has only previously been identified in two genital condylomas [113], and one malignant laryngeal lesion, the finding of this HPV genotype in eight OSCC samples has led to proposals that HPV 30 may have a proclivity to infect oesophageal mucosa [108].

This review underscores the highly variable results of HPV detection in OSCC, between different regions of China, for which diverse testing methodology may be a contributing factor. It is difficult to draw conclusions on which testing methods yield the highest and lowest rates of HPV detection since certain techniques such as FISH, SB and HB have been employed in very few studies and PCR which has been used in $76 \%$ of studies has also shown variable results between studies.

While general trends have reported higher HPV DNA detection rates in OSCC tissue from high incidence OSCC regions, the results of our review did not demonstrate this pattern. Thirty-four percent of all OSCC samples, sourced predominantly from the highest OSCC incidence region of Henan Province in eastern China, were positive for HPV DNA. However, we found that other high-incidence areas within China from the north-western counties in Xinjiang, to Guandong in the south-east, Hebei in the north and the central Province of Shaanxi have reported even higher rates of HPV DNA detection in OSCC tissue. Of note is a study carried out by Koshiol where only 3/267 OSCC samples tested using PCR, were positive for HPV [61]. This is one of the largest studies to be carried out and their result is particularly interesting as it has been conducted in Linxian, Henan province [30]. This result is not in keeping with the generally observed trends of high HPV detection rates in high-risk OSCC populations. Furthermore, the three studies in China which did not isolate HPV from OSCC, also recruited subjects residing in Henan province [52-54].

A recent study by Furrer $e t$ al. reported higher rates of HPV DNA detection in superficial oral scrapes compared to deep tissue biopsies from patients with oral cancer, in an Argentinian cohort [114]. They suggest that the site of specimen sampling is important in obtaining an accurate epidemiological picture on the HPV link to carcinogenesis. This hypothesis may be extended to oesophageal cancer and we therefore aimed to examine whether the location from which the oesophageal specimen was taken in OSCC patients, i.e. superficial scrapings or deep tissue biopsy, may have any correlation with HPV detection rate. We found that $35 \%$ of deep tissue biopsies and $41 \%$ of superficial scrapings from OSCC patients were HPV positive. This finding is consistent with results reported by Furrer et al.

With the growing evidence that the spectrum of HPVrelated malignancies may spread beyond cancers of the anogenital tract [1], the global health burden attributable to HPV continues to increase. As a result, there has been increasing pressure to make the HPV vaccines more widely available for males, thereby immunizing entire cohorts against the effects of this virus. If HPV plays a significant role in the aetiology of OSCC, the introduction of prophylactic HPV vaccines could have a public health impact in a nation such as China where OC is one of the leading causes of malignancy-related mortality.

In many developed countries public health funding for the prophylactic HPV vaccines is available for girls and young women prior to their sexual debut [115]. However, in China, there are currently no national programs for cervical cancer screening and a majority of women have never been screened. Consequently, at present, the prophylactic HPV vaccines have not been licensed for distribution in China.

Population-based surveys of Chinese women have been carried out recently to identify potential difficulties in the implementation of a prophylactic vaccination program in China $[116,117]$. One of the largest obstacles is the price of the vaccination, should government funding be insufficient to cover costs $[116,117]$. Prophylactic HPV vaccines are to date the most expensive vaccine developed with a retail price of US $\$ 120$ per dose of Gardasil $^{\circledR}$ (US \$360 for the complete course) excluding administrative costs [118]. Other problems include rural habitation with poor access to health services, cultural and religious barriers, personal attitudes and beliefs as well as limited knowledge of HPV and vaccination [116, 117]. However, the introduction of the Hepatitis B vaccine (HBV) to protect against hepatocellular carcinoma (HCC) in China may provide a template upon which the prophylactic HPV vaccination program can be modeled [117]. The first national HBV program was instigated in Taiwan in 1984 [119]. Over a 10 year period from 1984 to 1994, follow-up studies in children under the age of 15, demonstrated a reduction in HBsAg prevalence rates 
from $9 \cdot 8 \%$ to $1.3 \%$ [120]. A recent cross-sectional seroprevalence study by Chang et al. also reported a statistically significant reduction in incidence of HCC in a cohort of vaccinated children aged 6-19 years, compared to a comparable unvaccinated group [119].

From the results of this review, we determine that HPV DNA was found in over one third of OSCC tissue samples, compared to cervical cancer where HPV is responsible for the pathogenesis of $100 \%$ of lesions. Thus the impact of prophylactic vaccination would be considerably higher in cervical cancers than in OSCC, if a link exists. However, it is important to clarify any HPVOSCC association as even a $20 \%-30 \%$ rate of HPV infection as a causal co-factor would be significantly impacted by vaccination, particularly in a geographically targeted vaccination program.

\section{Conclusions}

We found that $36 \%$ of all OSCC samples and $28 \%$ of all healthy control samples tested from the Chinese population were positive for HPV DNA and the majority (11/16) of case-control and cross-sectional studies found a statistically significant association between HPV and OSCC.

The findings of this review are in line with the hypothesis that HPV detection rates are higher in superficial oesophageal cancer samples compared deep tissue specimens [114]. It may therefore be important to consider depth of tissue biopsy when interpreting epidemiological studies assessing HPV aetiology in malignancy.

Research carried out over the last 30 years has neither precluded nor established HPV as an aetiological factor in OSCC. The difficulty in determining a link may be due to several factors including 1) the poor methodological design and generally smaller sample sizes in a majority of studies. Only few case-control studies have ever been done, with the vast majority of studies on the subject being case-series, which are unable to adequately address the question of aetiology or risk factors. The fact that none of the identified case-control studies included statistical measures of association, even when data were collected to enable these measures, indicates the problem of poor study design; 2) the utilization of many different HPV detection methods with varying specificity and sensitivity ranges i.e. PCR with either general or consensus primers which identify different HPV genotypes, histological biopsy (HB), IHC, ISH, FISH, general primer (GP), consensus primer (CP), serological testing, hybrid capture; 3) inter-laboratory deviation on similar testing methodology; 4) utilization of various types of specimens i.e. balloon cell samples, OSCC tissue from resections or biopsies which may be either fresh or archival; 5) variation within tissue samples examined; 6) differences in histopathological classification and tissue storage i.e.
Iodine staining, paraffin samples; 7) the presence of potential co-factors (e.g. smoking, opium abuse, nutritional deficiencies, ingestion of nitrosamines and exposure to other industrial chemicals) which may be more important depending on geographical location, could act synergistically with HPV to promote infection of oesophageal tissue; 8) the possible "hit and run" mechanism proposed by Campo and modeled on observations of bovine papillomavirus type 4 (BPV-4) infection of bovine oesophageal tissue [121]; 9) genetic polymorphisms facilitating malignant transformation [122].

Despite the many factors which could be responsible for the high variability of results reported, it remains that an equal potential for inconsistency with similar variables existed in investigations carried out to establish the role of HPV in cervical and other HPV-related cancers, which have yielded more convincing results. Therefore, it may be inferred that if a link does exist between HPV and OSCC, it may be weaker than in other HPV-related cancer, or geographically varied and related to other cofactors [52].

A meta-analysis of existing case-control studies as well as further large-scale case-control studies with adequate statistical power are required to more meaningfully address whether a causal relationship between HPV and OSCC exists. The introduction of the prophylactic HPV vaccines has made it even more important to definitively determine the answer to this research question, particularly for countries such as China, where there is a significant cancer burden from oesophageal malignancy.

\section{REFERENCES}

[1] K. Syrjänen, “HPV and Oesophageal Carcinoma,” In: Papillomavirus Research: From Natural History to Vaccines and Beyond," Caister Academic Press, Norfolk, 2006, pp. 229-253.

[2] "Smokeless Tobacco and Some Tobacco-Specific N-Nitrosamines," IARC Monographs on the Evaluation of Carcinogenic Risks to Humans, Vol. 89, World Health Organisation and International Agency for Research on Cancer, Lyon, 2007.

[3] G. Brooks, K. Carroll, J. Butel, S. Morse and T. Mietzner, "Human Cancer Viruses,” In: M. A. Jawetz, Ed., Medical Microbiology, 24th Edition, The McGraw-Hill Companies, New York, 2007, pp. 597-600.

[4] P. Day and J. Schiller, "Early Events in the Papillomaviral Lifecycle,” In: C. M. Norfolk, Ed., Papillomavirus Research, Caister Academic Press, Norfolk, 2006, pp. 175-192.

[5] E. de Villiers, C. Fauquet, T. Broker, B. Hu and H. zur Hausen, "Classification of Papillomaviruses," Virology, Vol. 324, No. 1, 2004, pp. 17-27. doi:10.1016/j.virol.2004.03.033

[6] J. Ferlay, H. Shin, F. Bray, D. Forman, C. Mathers and D. 
Parkin, "GLOBOCAN 2008, Cancer Incidence and Mortality Worldwide: IARC Cancer Base,” No. 10, International Agency for Research on Cancer, Lyon, 2010.

[7] D. M. Parkin, F. Bray, J. Ferlay and P. Pisani, "Global Cancer Statistics, 2002,” CA: A Cancer Journal for Clinicians, Vol. 55, No. 2, 2005, pp. 74-108. doi:10.3322/canjclin.55.2.74

[8] H. zur Hausen, "Human Papillomaviruses in the Pathogenesis of Anogenital Cancer,” Virology, Vol. 184, No. 1, 1991, pp. 9-13. doi:10.1016/0042-6822(91)90816-T

[9] H. Romanczuk and P. Howley, "Disruption of Either the E1 or the E2 Regulatory Gene of Human Papillomavirus Type 16 Increases Viral Immortalization Capacity,” Proceedings of the National Academy of Sciences, Vol. 89, No. 7, 1992, pp. 3159-3163.

[10] L. F. Garrote, R. Herrero, R. M. O. Reyes, S. Vaccarella, J. L. Anta, L. Ferbeye, N. Munoz and S. Franceschi, "Risk Factors for Cancer of the Oral Cavity and Oro-Pharynx in Cuba," British Journal of Cancer, Vol. 85, No. 1, 2001, pp. 46-54. doi:10.1054/bjoc.2000.1825

[11] A. M. Hong, A. E. Grulich, D. Jones, C. S. Lee, S. M. Garland, T. A. Dobbins, J. R. Clark, G. B. Harnett, C. G. Milross, C. J. O’Brien, et al., "Squamous Cell Carcinoma of the Oropharynx in Australian Males Induced by $\mathrm{Hu}-$ man Papillomavirus Vaccine Targets,” Vaccine, Vol. 28, No. 19, 2010, pp. 3269-3272. doi:10.1016/j.vaccine.2010.02.098

[12] R. K. Aimee, J. A. Anthony, R. Daniel, E. G. Patti, R. Viscidi, S. G. Elizabeth, V. S. Keerti and L. Gillison Maura, "Oral Human Papillomavirus Infection in Adults Is Associated with Sexual Behavior and HIV Serostatus," The Journal of Infectious Diseases, Vol. 189, No. 4, 2004, pp. 686-698. doi:10.1086/381504

[13] C. H. Shiboski, B. L. Schmidt and R. C. K. Jordan, “Tongue and Tonsil Carcinoma,” Cancer, Vol. 103, No. 9, 2005, pp. 1843-1849. doi:10.1002/cncr.20998

[14] E. Smith, S. Johnson, T. Cripe, S. Pignatari and L. Turek, "Perinatal Vertical Transmission of Human Papillomavirus and Subsequent Development of Respiratory Tract Papillomatosis," Annals of Otology, Rhinology and Laryngology, Vol. 100, No. 6, 1991, pp. 478-483.

[15] F. Chang, S. Syrjanen, Q. Shen, L. Wang, D. Wang and K. Syrjänen, "Human Papillomavirus (HPV) Involvement in Esophageal Precancerous Lesions and Squamous Cell Carcinomas as Evidenced by Microscopy and Different DNA Techniques," Scandinavian Journal of Gastroenterology, Vol. 27, No. 7, 1992, pp. 553-563. doi:10.3109/00365529209000119

[16] M. Curado, B. Edwards, H. Shin, H. Storm, J. Ferlay, M. Heanue and P. Boyle, "Cancer Incidence in Five Continents,” International Agency for Research on Cancer, Lyon, 2007.

[17] S. Mudan and J. Kang, "Epidemiology and Clinical Presentation in Esophageal Cancer," In: S. Rankin, Ed., Carcinoma of the Esophagus, Cambridge University Press, New York, 2008, pp. 1-10.

[18] S. J. Spechler, "Squamous Cell Carcinoma of the Esophagus,” In: W. Scheppach, R. Bresalier and G. Tytgat, Eds.,
Gastrointestinal and Liver Tumors, Springer-Verlag, Berlin, 2004, pp. 5-11. doi:10.1007/978-3-642-18629-5_1

[19] Z. Wang, L. Tang, G. Sun, Y. Tang, Y. Xie, S. Wang, X. Hu, W. Gao, S. Cox and J.-S. Wang, "Etiological Study of Esophageal Squamous Cell Carcinoma in an Endemic Region: A Population-Based Case Control Study in Huaian, China,” BMC Cancer, Vol. 6, No. 1, 2006, p. 287. doi:10.1186/1471-2407-6-287

[20] D. Parkin, P. Pisani and J. Ferlay, "Estimates of Worldwide Incidence of 25 Major Cancers in 1990,” International Journal of Cancer, Vol. 80, No. 6, 1990, pp. 827 841.

doi:10.1002/(SICI)1097-0215(19990315)80:6<827::AIDIJC6>3.0.CO;2-P

[21] Esophageal Cancer. http://www.merckmanuals.com/home/sec09/ch131/ch131 c.html

[22] J. Ferlay, H. R. Shin, F. Bray, D. Forman, C. Mathers and D. M. Parkin, "Estimates of Worldwide Burden of Cancer in 2008: GLOBOCAN 2008,” International Journal of Cancer, Vol. 127, No. 12, 2010, pp. 2893-2917. doi:10.1002/ijc.25516

[23] P. Zhao, M. Dai, W. Chen and N. Li, "Cancer Trends in China,” Japanese Journal of Clinical Oncology, Vol. 40, No. 4, 2010, pp. 281-285. doi:10.1093/jico/hyp187

[24] H. Zhang, Z. Chen, J. Cheng, X. Zhu, W. Guo, A. Hu, Y. Du, Y. Zhou and Y. Wang, "The High Incidence of Esophageal Cancer in Parts of China May Result Primarily from Genetic Rather than Environmental Factors,” Diseases of the Esophagus, Vol. 23, No. 5, 2010, pp. 392397.

[25] D. He, D.-K. Zhang, K.-Y. Lam, L. Ma, H. Y.-S. Ngan, S. S. Liu and S.-W. Tsao, "Prevalence of HPV Infection in Esophageal Squamous Cell Carcinoma in Chinese Patients and Its Relationship to the p53 Gene Mutation,” International Journal of Cancer, Vol. 72, No. 6, 1997, pp. 959-964. doi:10.1002/(SICI)1097-0215(19970917)72:6<959::AIDIJC7>3.0.CO;2-O

[26] F. Kamangar, R. Malekzadeh, S. Dawsey and F. Saidi, "Esophageal Cancer in Northeastern Iran: A Review," Archives of Iranian Medicine, Vol. 10, No. 1, 2007, pp. 7082.

[27] S. Bai, S. Zhang and B. Li, "Primary Esophageal Adenocarcinoma," Chinese Journal of Oncology, Vol. 11, No. 5, 1989, pp. 383-385.

[28] S. S. Liyanage, E. Segelov, S. M. Garland, S. N. Tabrizi, H. Seale, P. J. Crowe, D. E. Dwyer, A. Barbour, A. T. Newall, A. Malik, et al., "The Role of Human Papillomaviruses in Esophageal Squamous Cell Carcinoma," Asia-Pacific Journal of Clinical Oncology, Vol. 9, No. 1, 2013, pp. 12-28.

[29] K. Syrjänen, "HPV Infections and Oesophageal Cancer," Journal of Clinical Pathology, Vol. 55, No. 10, 2002, pp. 721-728. doi:10.1136/jcp.55.10.721

[30] L. Ke, "Mortality and Incidence Trends from Esophagus Cancer in Selected Geographic Areas of China Circa 1970-90,” International Journal of Cancer, Vol. 102, No. 


\section{3, 2002, pp. 271-274. doi:10.1002/ijc.10706}

[31] Y. Qin, L. Wang, Z. Fan, D. Kwong and X. Guan, “Comparative Genomic Hybridization Analysis of Genetic Aberrations Associated with Development of Esophageal Squamous Cell Carcinoma in Henan, China,” World Journal of Gastroenterology, Vol. 14, No. 12, 2008, pp. 18281835. doi:10.3748/wjg.14.1828

[32] C. Yang, "Research on Esophageal Cancer in China: A Review," Cancer Research, Vol. 40, No. 8, 1980, pp. 26332644.

[33] X. Castellsagué, N. Muñoz, E. De Stefani, C. Victora, R. Castelletto, P. Rolón and M. Quintana, "Independent and Joint Effects of Tobacco Smoking and Alcohol Drinking on the Risk of Esophageal Cancer in Men and Women,” International Journal of Cancer, Vol. 82, No. 5, 1999, pp. 657-664.

doi:10.1002/(SICI)1097-0215(19990827)82:5<657::AIDIJC7>3.0.CO;2-C

[34] C. Han, G. Qiao, N. L. Hubbert, L. Li, C. Sun, Y. Wang, M. Yan, D. Xu, Y. Li, D. R. Lowy, et al., "Serologic Association between Human Papillomavirus Type 16 Infection and Esophageal Cancer in Shaanxi Province, China,” Journal of the National Cancer Institute, Vol. 88, No. 20, 1996, pp. 1467-1471. doi:10.1093/jnci/88.20.1467

[35] J. Lagergren, Z. Wang, R. Bergstrom, J. Dillner and O. Nyren, "Human Papillomavirus Infection and Esophageal Cancer: A Nationwide Seroepidemiologic Case-Control Study in Sweden," Journal of the National Cancer Institute, Vol. 91, No. 2, 1999, pp. 156-162. doi:10.1093/jnci/91.2.156

[36] C. Bosetti, C. La Vecchia, R. Talamini, L. Simonato, P. Zambon, E. Negri, D. Trichopoulos, P. Lagiou, R. Bardini and S. Franceschi, "Food Groups and Risk of Squamous Cell Esophageal Cancer in Northern Italy,” Journal of Cancer, Vol. 87, No. 2, 2000, pp. 289-294.

[37] G. Launoy, C. Milan, N. E. Day, M. P. Pienkowski, M. Gignoux and J. Faivre, "Diet and Squamous-Cell Cancer of the Oesophagus: A French Multicentre Case-Control Study," International Journal of Cancer, Vol. 76, No. 1, 1998, pp. 7-12. doi:10.1002/(SICI)1097-0215(19980330)76:1<7::AID-IJ $\mathrm{C} 2>3.0 . \mathrm{CO} ; 2-4$

[38] A. Ghavamzadeh, A. Moussavi, M. Jahani, M. Rastegarpanah and M. Iravani, "Esophageal Cancer in Iran,” Seminars in Oncology, Vol. 28, No. 2, 2001, pp. 153-157. doi:10.1016/S0093-7754(01)90086-7

[39] F. Islami, P. Boffetta, J.-S. Ren, L. Pedoeim, D. Khatib and F. Kamangar, "High-Temperature Beverages and Foods and Esophageal Cancer Risk-A Systematic Review," International Journal of Cancer, Vol. 125, No. 3, 2009, pp. 491-524. doi:10.1002/ijc.24445

[40] F. Chang, S. Syrjänen, L. Wang and K. Syrjänen, "Infectious Agents in the Etiology of Esophageal Cancer," Gastroenterology, Vol. 103, No. 4, 1992, pp. 1335-1348.

[41] V. Del, M. White, S. Enam, S. Oviedo, M. Bromer, R. Thomas, H. Parkman and K. Khalili, "Detection of JC Virus DNA Sequences and Expression of Viral T Antigen and Agnoprotein in Esophageal Carcinoma,” Cancer, Vol.
103, No. 3, 2005, pp. 516-527. doi:10.1002/cncr.20806

[42] M. Poljak, A. Cerar and K. Seme, "Human Papillomavirus Infection in Esophageal Carcinomas: A Study of 121 lesions Using Multiple Broad-Spectrum Polymerase Chain Reactions and Literature Review," Human Pathology, Vol. 29, No. 3, 1998, pp. 266-271. doi:10.1016/S0046-8177(98)90046-6

[43] M. Selgrad, P. Malfertheiner, L. Fini, A. Goel, C. R. Boland and L. Ricciardiello, "The Role of Viral and Bacterial Pathogens in Gastrointestinal Cancer,” Journal of Cellular Physiology, Vol. 216, No. 2, 2008, pp. 378-388. doi:10.1002/jcp.21427

[44] M. Sur and K. Cooper, "The Role of the Human Papillomavirus in Esophageal Cancer,” Pathology, Vol. 30, No. 4, 1998, pp. 358-354. doi:10.1080/00313029800169616

[45] M. Tandi Matsha, R. Erasmus, A. Kafuko, D. Mugwanya, A. Stepien and M. I. Parker, "Human Papillomavirus Associated with Cancer," Journal of Clinical Pathology, Vol. 55, No. 8, 2002, pp. 587-590. doi:10.1136/jcp.55.8.587

[46] K. Syrjänen, "Histological Changes Identical to Those of Condylomatous Lesions Found in Esophageal Squamous Cell Carcinomas," Archiv für Geschwulstforschung, Vol. 52, No. 4, 1982, pp. 283-292.

[47] L. Li, "Morbidity and Mortality of Malignant Tumours in Some Chinese Cities and Counties,” First Edition, People’s Medical Publishing House, Beijing, 2007.

[48] C. Hennekens and J. Buring, "Case-Control Studies," In: S. M. Boston, Ed., Epidemiology in Medicine, First Edition, Little, Brown and Company, Toronto, 1987, pp. 132152.

[49] R. Friis and T. Sellers, "Study Designs: Ecologic, CrossSectional, Case-Control,” In: Epidemiology for Public Health Practice, Fourth Edition, Jones and Bartlett Publishers, LLC, Sudbury, 2009, pp. 262-275.

[50] A. Dean, T. Arner, G. Sunki, R. Friedman, M. Lantinga, S. Sangam, J. Zubieta, K. Sullivan, K. Brendel, Z. Gao, et al., "Epi Info, a Database and Statistics Program for Public Health Professionals,” 3.5.3 Edition, Centres for Diseas Control and Prevention (CDC), Atlanta, 2007.

[51] M. Mori, R. Shimono, T. Inoue, H. Kuwano, K. Sugimachi and R. Zhang, "Papillomavirus and Esophageal Cancer in the Japanese and Chinese,” The American Journal of Gastroenterology, Vol. 84, No. 9, 1989, pp. 11261127.

[52] G.-F. Gao, M. J. Roth, W.-Q. Wei, C. C. Abnet, F. Chen, N. Lu, F.-H. Zhao, X.-Q. Li, G.-Q. Wang, P. R. Taylor, et al., "No Association between HPV Infection and the Neoplastic Progression of Esophageal Squamous Cell Carcinoma: Result from a Cross-Sectional Study in a HighRisk Region of China,” International Journal of Cancer, Vol. 119, No. 6, 2006, pp. 1354-1359.

[53] S. Lu, F. Luo and H. Li, "Detection of Human Papillomavirus in Esophageal Squamous Cell Carcinoma and Adjacent Tissue Specimens in Linxian,” Chinese Journal of Oncology, 1995, Vol. 17, No. 5, pp. 321-324.

[54] C.-S. Song, J.-H. Cui, Z.-P. Wu, G. Yang and J.-J. Tan, "Relationship between Detection pf Human Papilloma 
Virus Genotypes and Esophageal Squamous Cell Carcinoma," Chinese Journal of Cancer Prevention and Treatment, Vol. 16, No. 5, 2009, pp. 329-331.

[55] B. Cao, X. Tian, Y. Li, P. Jiang, T. Ning, H. Xing, Y. Zhao, C. Zhang, X. Shi, D. Chen, et al., "LMP7/TAP2 Gene Polymorphisms and HPV Infection in Esophageal Carcinoma Patients from a High Incidence Area in China," Carcinogenesis, Vol. 26, No. 7, 2005, pp. 12801284.

[56] B. Chen, "Polymerase Chain Reaction in Detection of Human Papillomavirus DNA in Esophageal Carcinoma,” Chinese Medical Journal, Vol. 73, No. 11, 1993, pp. 667669.

[57] B. Chen, H. Yin and N. Dhurandhar, "Detection of Human Papillomavirus DNA in Esophageal Squamous Cell Carcinomas by the Polymerase Chain Reaction Using General Consensus Primers,” Human Pathology, Vol. 25, No. 9, 1994, pp. 920-923.

[58] M. Dai, W. Zhang, G. Clifford, T. Gheit, B. He, K. Michael, T. Waterboer, P. Hainaut, M. Tommasino and S. Franceschi, "Human Papillomavirus Infection among 100 Oesophageal Cancer Cases in the People's Republic of China,” International Journal of Cancer, Vol. 121, No. 6, 2007, pp. 1396-1398.

[59] E. de Villiers, D. Lavergne, F. Chang, K. Syrjänen, P. Tosi, M. Cintorino, R. Santopietro and S. Syrjänen, “An Interlaboratory Study to Determine the Presence of $\mathrm{Hu}$ man Papillomavirus DNA in Esophageal Carcinoma from China,” International Journal of Cancer, Vol. 81, No. 2, 1999, pp. 225-228.

[60] D. He, S. Tsao and H. Bu, "Human Papillomavirus Infection and Esophageal Squamous Cell Carcinoma,” Chinese Journal of Pathology, Vol. 25, No. 6, 1996, pp. 351-354.

[61] J. Koshiol, W. Q. Wei, A. R. Kreimer, W. Chen, P. Gravitt, J. S. Ren, C. C. Abnet, J. B. Wang, F. Kamangar, D. M. Lin, et al., "No Role for Human Papillomavirus in Esophageal Squamous Cell Carcinoma in China,” International Journal of Cancer, Vol. 127, No. 1, 2010, pp. 93-100.

[62] D. Lavergne and E. M. de Villiers, "Papillomavirus in Esophageal Papillomas and Carcinomas," International Journal of Cancer, Vol. 80, No. 5, 1999, pp. 681-684.

[63] S. Li, Y. Li, L. Wang, X. Wu, L. Zhou, X. Zhao, H. Liu and Y. Zeng, "Detection of Human Papillomavirus in Tissues of Esophageal Carcinomas by Polymerase Chain Reaction,” Chinese Journal of Experimental and Clinical Virology, Vol. 22, No. 4, 2008, pp. 251-253.

[64] T. Li, Z. M. Lu, M. Guo, Q. J. Wu, K. N. Chen, H. P. Xing, Q. Mei and Y. Ke, "p53 Codon 72 Polymorphism $(\mathrm{C} / \mathrm{G})$ and the Risk of Human Papillomavirus-Associated Carcinomas in China," Cancer, Vol. 95, No. 12, 2002, pp. 2571-2576.

[65] T. Li, Z.-M. Lu, K.-N. Chen, M. Guo, H.-P. Xing, Q. Mei, H.-H. Yang, J. F. Lechner and Y. Ke, "Human Papillomavirus Type 16 is an Important Infectious Factor in the High Incidence of Esophageal Cancer in Anyang Area of China," Carcinogenesis, Vol. 22, No. 6, 2001, pp. 929934.
[66] P. Liao, J. Qin, T. Zeng, F. Li, J. Cai and L. He, “A 1:2 Matched Case-Control Study on the Interaction of HPV16E6 and HLA-DR9 Allele to Esophageal Cancer in Kazakh Ethnicity, Xinjiang," Chinese Journal of Epidemiology, Vol. 30, No. 9, 2009, pp. 951-954.

[67] M. Liu, H. Zeng, X. Zhang, J. Zhu, J. Huang, X. Zhang and M. Xia, "Study on Human Papillomavirus Infection and Loss of Heterozygosity of Microsatellite in Esophageal Cancer," Chinese Journal of Epidemiology, Vol. 28, No. 12, 2007, pp. 1203-1206.

[68] Y. Liu, X. Li, G. Jin, X. Yan, J. Yang, J. Wang, Y. Li, F. Wang and X. Zhang, "HPV Detection and FHIT Expression in Esophageal Squamous Carcinoma from High Incidence Area in Cixian County," Chinese Journal of Cancer, Vol. 22, No. 5, 2003, pp. 492-495.

[69] X. Lu, Y. Zhang, R. Lin, X. Liang, Y. Zhang, X. Wang, Y. Zhang, Y. Wang and H. Wen, "p53 Polymorphism in Human Papillomavirus-Associated Kazakh’s Esophageal Cancer in Xinjiang, China,” World Journal of Gastroenterology, Vol. 10, No. 19, 2004, pp. 2775-2778.

[70] X. M. Lu, S. Monnier-Benoit, L. Z. Mo, S. Y. Xu, J. L. Prétet, Z. Liu, D. A. Vuitton and C. Mougin, "Human Papillomavirus in Esophageal Squamous Cell Carcinoma of the High-Risk Kazakh Ethnic Group in Xinjiang, China,” European Journal of Surgical Oncology, Vol. 34, No. 7, 2008, pp. 765-770.

[71] Z. Lu, K. Chen and M. Guo, "Detection of HPV in Human Oesophageal Cancer in High Incidence Area and Its Correlation with p53 Expression," Chinese Journal of Oncology, Vol. 23, No. 3, 2001, pp. 220-223.

[72] D. Peixoto Guimaraes, S. Hsin Lu, P. Snijders, R. Wilmotte, R. Herrero, G. Lenoir, R. Montesano, C. J. L. M. Meijer, J. Walboomers and P. Hainaut, "Absence of Association between HPV DNA, TP53 Codon 72 Polymorphism, and Risk of Oesophageal Cancer in a High-Risk Area of China," Cancer Letters, Vol. 162, No. 2, 2001, pp. 231-235.

[73] Z. Y. Shen, S. P. Hu, L. C. Lu, C. Z. Tang, Z. S. Kuang, S. P. Zhong and Y. Zeng, "Detection of Human Papillomavirus in Esophageal Carcinoma," Journal of Medical Virology, Vol. 68, No. 3, 2002, pp. 412-416.

[74] K. Shuyama, A. Castillo, F. Aguayo, Q. Sun, N. Khan, C. Koriyama and S. Akiba, "Human Papillomavirus in Highand Low-Risk Areas of Oesophageal Squamous Cell Carcinoma in China,” British Journal of Cancer, Vol. 96, No. 10, 2007, pp. 1554-1559.

[75] H. Si, S. Tsao, C. Poon and A. Cheung, "Human Papillomavirus Infection and Loss of Heterozygosity in Esophageal Squamous Cell Carcinoma," Cancer Letter, Vol. 213, No. 2, 2004, pp. 231-239.

[76] H. X. Si, S. W. Tsao, C. S. P. Poon, L. D. Wang, Y. C. Wong and A. L. Cheung, "Viral Load of HPV in Esophageal Squamous Cell Carcinoma,” International Journal of Cancer, Vol. 103, No. 4, 2003, pp. 496-500.

[77] L. Suzuk, A. Noffsinger, Y. Hui and C. Fenoglio-Preiser, "Detection of Human Papillomavirus in Esophageal Squamous Cell Carcinoma,” Cancer, Vol. 78, No. 4, 1996, pp. 704-710. 
[78] W. Xu, L. Zhang, Z. Lu, J. Li, Y. Ke and G. Xu, “Detection of Human Papillomavirus Type 16 E6 mRNA in Carcinomas of the Upper Digestive Tract," Chinese Medical Journal, Vol. 83, No. 21, 2003, pp. 1910-1914.

[79] X. Zhao, S. Li, Y. Li, X. Wang, Y. Li, X. Wu, L. Zhou, H. Liu and Y. Zeng, "Detection of Human Papillomavirus in Esophageal Carcinoma Tissues from Baoding City of Hebei Province," Chinese Journal of Experimental and Clinical Virology, Vol. 23, No. 2, 2009, pp. 91-93.

[80] X. Zhou, M. Guo, L. Quan, W. Zhang, Z. Lu, Q. Wang, Y. Ke and N. Xu, "Detection of Human Papillomavirus in Chinese Esophageal Squamous Cell Carcinoma and Its Adjacent Normal Epithelium,” World Journal of Gastroenterology, Vol. 9, No. 6, 2003, pp. 1170-1173.

[81] Y. Zhou, Y. Pan, S. Zhang, X. Shi, T. Ning and Y. Ke, "Increased Phosphorylation of p70 S6 Kinase Is Associated with HPV16 Infection in Cervical Cancer and Esophageal Cancer,” British Journal of Cancer, Vol. 97, No. 2, 2007, pp. 218-222.

[82] F. Chang, S. Syrjänen, Q. Shen, M. Cintorino, R. Santopietro, P. Tosi and K. Syrjänen, "Detection of HPV DNA in Esophageal Squamous Cell Carcinoma from the High Incidence Area of Linxian, China," Scandinavian Journal of Gastroenterology, Vol. 35, No. 2, 2000, pp. 123-130.

[83] J. Chen, Y. Zhou, J. Li and P. Wu, "Effect of HPV16 Infection on the Esophageal Carcinoma and the Gene Expressions of p53 and p16," China Journal of Laboratory Diagnosis, Vol. 8, No. 2, 2004, pp. 182-185.

[84] L. Chen, L. Yang, Z. Sun, H. Zhang, T. Ren, B. Chang, L. Pang, Y. Qi, H. Li, J. Jiang, et al., "Correlation between HPV16 and Esophageal Cancer of Kazakh People in Xinjiang," Journal of Nongken Medicine, Vol. 30, No. 1, 2008, pp. 15-22.

[85] M. Duan, R. Tian and X. Cao, "The Relationship between HPV and Esophageal Carcinoma,” Journal of Practical Medical Techniques, Vol. 14, No. 1A, 2005, pp. 11-12.

[86] L. Y. Gu, L. M. Sai, X. L. Hou, G. Chen, F. Li, C. X. Liu, Y. Z. Chen, B. Chang, L. Yang, W. H. Liang, et al., "Correlation between HPV16 Infection and Esophageal Carcinoma of Kazakh People in Xinjiang," China Oncology, Vol. 21, No. 9, 2010, pp. 668-672.

[87] B. He, G. Duan and L. Cai, "Study on the Relationship between High Risk HPV Infection and p53 Polymorphisms in Esophageal Cancer," Strait Journal of Preventive Medicine, Vol. 13, No. 3, 2007, pp. 8-10.

[88] M. Liu, X. Zhang, W. Fu, J. Yang and X. Zhu, "Study of Association between HPV Infection and Squamous Cell Carinoma as well as Dysplastic Tissue of the Oesophagus,” China Journal of Modern Medicine, Vol. 18, No. 14, 2008, pp. 2084-2086.

[89] W. Liu, X. Jiang, M. Zhang and Z. Zhang, "The Relationship between HPV16 and Expression of Cyclooxygenase-2, P53 and Their Prognostic Roles in Esophageal Squamous Cell Carcinoma,” European Journal of Gastroenterology \& Hepatology, Vol. 22, No. 1, 2010, pp. 67-74. doi:10.1097/MEG.0b013e32832c7e76

[90] X. Wang, X. Tian, F. Liu, Y. Zhao, M. Sun, D. Chen, C.
Lu, Z. Wang, X. Shi, Q. Zhang, et al., "Detection of HPV DNA in Esophageal Cancer Specimens from Different Regions and Ethnic Groups: A Descriptive Study,” BMC Cancer, Vol. 10, No. 1, 2010, p. 19. doi:10.1186/1471-2407-10-19

[91] S. Zhou, I. Sheyhidin, T. Yang, L. Zhang, A. Hasim, X. Lu, M. Niyaz and T. Liu, "Relationship between Human Papillomavirus 16 and Esophageal Squamous Cell Carcinoma in Uygur Population in Xinjiang Uygur Autonomous Region," World Chinese Journal of Digestology, 2009, Vol. 17, No. 31, pp. 3214-3217.

[92] M.-C. Cui, Y. Li, X. He, X.-L. Wang, L.-D. Wang and H.-S. Liu, "Study of Human Papillomavirus in Biospy Tissue Specimens of Esophageal Carcinomas in Linzhou City," Chinese Journal of Experimental and Clinical Virology, Vol. 25, No. 1, 2011, pp. 39-41.

[93] A. Goto, C.-P. Li, S. Ota, T. Niki, Y. Ohtsuki, S. Kitajima, S. Yonezawa, C. Koriyama, S. Akiba, H. Uchima, et al., "Human Papillomavirus Infection in Lung and Esophageal Cancers: Analysis of 485 Asian Cases," Journal of Medical Virology, Vol. 83, No. 8, 2011, pp. 1383-1390. doi:10.1002/jmv.22150

[94] H. Kawaguchi, S. Ohno, K. Araki, M. Miyazaki, H. Saeki, M. Watanabe, S. Tanaka and K. Sugimachi, "p53 Polymorphism in Human Papillomavirus-Associated Esophageal,” Cancer Cancer Researchearch, Vol. 60, 2000, pp. 2753-2755.

[95] Q. Ma, H. Jiang, Y. Feng, X. Wang, Y. Zhou, K. Liu and Z. KJia, "Detection of Human Papillomavirus DNA in Squamus Cell Carcinoma of the Esophagus,” World Chinese Journal of Digestology, Vol. 8, No. 11, 2000, pp. 1218-1224.

[96] L. Yang, L. Chen, Z.-Z. Sun, H.-Y. Zhang, X.-Y. Tian, Y. Qi, J. Zhu, L. Yang, J.-M. Qin and F. Li, “Analysis of Human Papillomavirus DNA Infection in Kazakh Esophageal Carcinomas in Xinjiang Province,” World Chinese Journal of Digestology, Vol. 15, No. 9, 2007, pp. 2114-2119.

[97] G. Ding, L. Wang, S.-H. Li, C. Feng, Y.-R. Zhang, J.-L. Ren, Z. Fan, R. Wang, X. He and T. Guo, "Correlation between Papillomavirus Infection and Esophageal Squamous Cell Carcinoma and Gastric Adenocarcinoma in High-Incidence Area of Henan Province," Chinese Journal of Cancer Prevention and Treatment, Vol. 16, No. 4, 2009, pp. 252-255.

[98] G.-C. Ding, J.-L. Ren, F.-B. Chang, J.-L. Li, L. Yaun, X. Song, S.-L. Zhou, T. Guo, Z.-M. Fa, Y. Zeng, et al., "Human Papillomavirus DNA and p16 INK4A Expression in Concurrent Esophageal and Gastric Cardia Cancers," World Journal of Gastroenterology, Vol. 16, No. 46, 2010, pp. 5901-5906. doi:10.3748/wjg.v16.i46.5901

[99] D. Zhang, Q. Zhang, L. Zhou, L. Huo, Y.-R. Zhang, Z. Shen and Y. Zhu, "Comparison of Prevalence, Viral Load, Physical Status and Expression of Human Papillimavirus-16, -18 and -58 in Esophageal and Cervical Cancer: A Case-Control Study,” BMC Cancer, Vol. 10, No. 650, 2010, p. 650. doi:10.1186/1471-2407-10-650

[100] C. Xu, X. Qian, X. Zhou, Q. Zhao and Y. Li, “Expression 
HPV 16-E6 and E7 Oncoproteins in Squamous Cell Carcinoma Tissues of Esophageal Cancer and Non-Cancer Tissues," Chinese Journal of Oncology, Vol. 23, No. 2, 2004, pp. 165-168.

[101] J. Liu, S. Qin and W. Zhang, "Relationship between HPVE6 p53 Protein and Esophageal Squamous Cell Carcinoma," World Chinese Journal of Digestology, Vol. 8, No. 5, 2000, pp. 494-496.

[102] F. Chang, S. Syrjänen, Q. Shen, H. Ji and K. Syrjänen, "Human Papillomavirus (HPV) DNA in Esophageal Precancer Lesions and Squamous Cell Carcinomas from China,” International Journal of Cancer, Vol. 45, No. 1, 1990, pp. 21-25. doi:10.1002/ijc.2910450106

[103] Y. Li, G. Huang, H. Xiao, Y. Huang, T. Mao and W. Deng, "The Status of Human Papillomavirus 16 DNA in the Tissues of Human Esophagus Carcinoma,” Journal of West China University of Medical Sciences, Vol. 22, No. 2, 1991, pp. 157-160.

[104] J. Li, Y. Zhang and D. Gao, "Study on the Interrelationship between Human Papilloma Virus Infection and Langerhans Cells in Carcinogenesis of Esophagus," Chinese Journal of Pathology, Vol. 25, No. 2, 1996, pp. 83-85.

[105] F. Chang, S. Syrjänen, L. Wang, Q. Shen and K. Syrjänen, "p53 Overexpression and Human Papillomavirus (HPV) Infection in Oesophageal Squamous Cell Carcinomas Derived from a High-Incidence Area in China," AntiCancer Researchearch, Vol. 17, No. 1B, 1997, pp. 709-715.

[106] L. Zhu, X. Su, K. Chen, R. Yang, H. Xing, J. Cui and Y. Ke, "Detection Rate of Human Papillomavirus-16 in Esophageal Squamous Cell Carcinoma from Different Chinese Populations,” Chinese Journal of Cancer, Vol. 24, No. 7, 2005, pp. 870-873.

[107] P. Yao, G. Li, J. Li, H. Xia, X. Yang, H. Huang, Y. Fu, R. Wang, X. Wang and J. Sha, "Evidence of Human Papilloma Virus Infection and Its Epidemiology in Esophageal Squamous Cell Carcinoma," World Journal of Gastroenterology, Vol. 12, No. 9, 2006, pp. 1352-1355.

[108] F. Chang, S. Syrjänen, Q. Shen, L. Wang and K. Syrjänen, "Screening for Human Papillomavirus Infections in Esophageal Squamous Cell Carcinomas by in Situ Hybridization,” Cancer, Vol. 72, No. 9, 1993, pp. 2525-2530. doi:10.1002/1097-0142(19931101)72:9<2525::AID-CNC R2820720902>3.0.CO;2-L

[109] P. Zhou, X.-Z. Wang, Z.-L. Zhu, J. Liu and X. Li, “Expression of HPV16/18 in Esophageal Squamous Carcinoma in Taixing Area," Journal of China Medical University, Vol. 40, No. 2, 2011, pp. 172-174.

[110] L. Jin and Q. Cao, "Expression and Effect of Human Papillomavirus in Esophageal Carcinoma," Chinese Journal of Gastroenterology, Vol. 12, No. 1, 2007, pp. 36-39.

[111] Z. X. Qi, X. Xu, B. Zhang, M. Du, H. Yang, L. Zheng, J. $\mathrm{Li}$ and Z. Shen, "Relationship between HPV 16/18 and p53, p21WAF1, MDM2, Ki67 and Cyclin D1 Expression in Esophageal Squamous Cell Carcinoma: Comparative Study by Using Tissue Microarray Technology,” Experimental Oncology, Vol. 28, No. 3, 2006, pp. 235-240.

[112] P. O. Fidalgo, M. L. Cravo, P. P. Chaves, C. N. Leitão and F. C. Mira, "High Prevalence of Human Papilloma- virus in Squamous Cell Carcinoma and Matched Normal Esophageal Mucosa. Assessment by Polymerase Chain Reaction,” Cancer, Vol. 76, No. 9, 1995, pp. 1522-1528. doi:10.1002/1097-0142(19951101)76:9<1522::AID-CNC $\underline{\text { R2820760904>3.0.CO;2-3 }}$

[113] T. Kahn, E. Schwarz and H. zur Hausen, "Molecular Cloning and Characterization of the DNA of a New Human Papillomavirus (HPV 30) from a Laryngeal Carcinoma,” International Journal of Cancer, Vol. 37, No. 1, 1986, pp. 61-65. doi:10.1002/ijc.2910370111

[114] V. Furrer, M. Benitez, M. Furnes, H. Lanfranchi and N. Modesti, "Biopsy vs. Superficial Scraping: Detection of Human Papillomavirus 6, 11, 16, and 18 in Potentially Malignant and Malignant Oral Lesions,” Journal of Oral Pathology \& Medicine, Vol. 35, No. 6, 2006, pp. 338344. doi:10.1111/j.1600-0714.2006.00423.x

[115] HPV Vaccination, "Should it be Recommended or Required?” Rapid Public Health Policy Response Project, Jacobs Institute of Women's Health, School of Public Health and Health Services, The George Washington University, Washington DC, 2007.

[116] J. Li, L. Li, J. Ma, L. Wei, M. Niyazi, C. Li, A. Xuh, J. Wanga, H. Lianga, J. Belinsoni, et al., "Knowledge and Attitudes about Human Papillomavirus (HPV) and HPV Vaccines among Women Living in Metropolitan and Rural Regions of China," Vaccine, Vol. 27, No. 8, 2009, pp. 1210-1215. doi:10.1016/j.vaccine.2008.12.020

[117] J. Shi, Y. Qiao, J. Smith, B. Dondog, Y. Bao, M. Dai, G. Clifford and S. Franceschi, "Epidemiology and Prevention of Human Papillomavirus and Cervical Cancer in China and Mongolia," Vaccine, Vol. 26S, No. 12, 2008, pp. M53-M59. doi:10.1016/j.vaccine.2008.05.009

[118] http://www.merck.com/newsroom/press_releases/product/ 2006_0608.html

[119] M. Chang, S. You, C. Chen, C. Liu, C. Lee, S. Lin, H. Chu, T. Wu, S. Yang, H. Kuo, et al., "Decreased Incidence of Hepatocellular Carcinoma in Hepatitis B Vaccinees: A 20-Year Follow-Up Study," Journal of the National Cancer Institute, Vol. 101, 2009, pp. 1348-1355. doi:10.1093/jnci/djp288

[120] H. Chen, M. Chang, Y. Ni, H. Hsu, P. Lee, C. Lee and D. Chen, "Seroepidemiology of Hepatitis B Virus Infection in Children: Ten Years of Mass Vaccination in Taiwan," Journal of the American Medical Association, Vol. 276, No. 11, 1996, pp. 906-908. doi:10.1001/jama.1996.03540110060032

[121] M. Campo, “Papillomas and Cancer in Cattle,” Cancer Survivors, Vol. 6, No. 1, 1987, pp. 39-54.

[122] D. Xing, W. Tan and D. Lin, "Genetic Polymorphisms and Susceptibility to Esophageal Cancer among Chinese Population,” Oncology Reports, Vol. 10, No. 5, 2003, pp. 1615-1623.

[123] F. Chang, S. Syrjänen, Q. Shen, M. Cintorino, R. Santopietro, P. Tosi and K. Syrjänen, "Evaluation of HPV, CMV, HSV and EBV in Esophageal Squamous Cell Carcinomas from a High-Incidence Area of China," AntiCancer Research, Vol. 20, No. 5C, 2000, pp. 3935-3940.

[124] X. Wang, X. Tian, F. Liu, Y. Zhao, M. Sun, D. Chen, C. 
Lu, Z. Wang, X. Shi, Q. Zhang, et al., "Detection of HPV

DNA in Esophageal Cancer Specimens from Different

Regions and Ethnic Groups: A Descriptive Study,” BMC
Cancer, Vol. 10, No. 19, 2010, p. 19.

HCC: Hepatocellular carcinoma

HB: Histological biopsy

HPV: Human papillomavirus

IHC: Immunohistochemistry

ISH: In situ hybridisation

IARC: International Agency on Research on Cancer OC: Oesophageal cancer

OSCC: Oesophageal squamous cell carcinoma

PCR: Polymerase chain reaction 